\title{
Swift J164449.3+573451 and Swift J2058.4+0516: Black hole mass estimates for tidal disruption event sources
}

\author{
Elena Seifina ${ }^{1,2}$, Lev Titarchuk ${ }^{3,4}$, and Enrico Virgilli ${ }^{3}$ \\ 1 LAPTh, 74941 Annecy-le-Vieux, France \\ e-mail: seifina@lapth.cnrs.fr \\ 2 Lomonosov Moscow State University/Sternberg Astronomical Institute, Universitetsky Prospect 13, 119992 Moscow, Russia \\ e-mail: seif@sai.msu.ru \\ 3 Dipartimento di Fisica, Università di Ferrara, via Saragat 1, 44122 Ferrara, Italy \\ e-mail: [titarchuk;virgilli]@fe.infn.it \\ ${ }^{4}$ National Research Nuclear University, MEPhI (Moscow Engineering Physics Institute), Moscow, Russia
}

Received 25 March 2017 / Accepted 7 July 2017

\begin{abstract}
A tidal disruption event (TDE) is an astronomical phenomenon in which a previously dormant black hole (BH) destroys a star passing too close to its central part. We analyzed the flaring episode detected from the TDE sources, Swift J164449.3+573451 and Swift J2058.4+0516 (hereafter Swift J1644+57 and Swift J2058+05, respectively) using RXTE, Swift and Suzaku data. The spectra are well fitted by the so called Bulk Motion Comptonization model for which the best-fit photon index $\Gamma$ varies from 1.1 to 1.8. We have firmly established the saturation of the photon index versus mass accretion rate at $\Gamma_{\text {sat }}$ about $1.7-1.8$. The saturation of $\Gamma$ is usually identified as a signature of a BH now established in Swift J1644+57 and Swift J2058+05. In Swift J1644+57 we found the relatively low $\Gamma_{\text {sat }}$ values which indicate a high electron (plasma) temperature, $k T_{\mathrm{e}} \sim 30-40 \mathrm{keV}$. This is also consistent with high cutoff energies, $E_{\text {cut }} \sim 60-80 \mathrm{keV}$ found using best fits of the RXTE spectra. Swift J2058+05 shows a lower electron temperature, $k T_{\mathrm{e}} \sim 4-10 \mathrm{keV}$ than that for Swift J1644+57. For the BH mass estimate we used the scaling technique taking the Galactic BHs, GRO J1655-40, GX 339-4, Cyg X-1 and 4U 1543-47 as reference sources and found that the BH mass in Swift J1644+57 is $M_{\mathrm{BH}} \geq 7 \times 10^{6} M_{\odot}$ assuming the distance to this source of $1.5 \mathrm{Gpc}$. For Swift J2058+05 we obtain $M_{\mathrm{BH}} \geq 2 \times 10^{7} M_{\odot}$ assuming the distance to this source of $3.7 \mathrm{Gpc}$. We have also found that the seed (disk) photon temperatures are quite low, of order of $100-400 \mathrm{eV}$, in both of the sources, which are consistent with the estimated BH masses.
\end{abstract}

Key words. accretion, accretion disks - galaxies: individual: Swift J164449.3+573451 - galaxies: individual: Swift J2058.4+0516 galaxies: general - radiation mechanisms: general - black hole physics

\section{Introduction}

Rees (1988, hereafter R88), suggested that among bright X-ray flares that continue for a few years, some can be caused by tidal gravitational disruption of a star which passed too close to a super massive black hole (SMBH). If a star passes within the tidal radius of a black hole then the gravity rips the star apart (R88). As the stellar remnant approaches a black hole (BH), its gravitational potential energy is converted into heat through viscous effects. Some of the debris can be ejected, while the remaining part can be ingested by a central SMBH. In this case the accretion flow reaches a temperature of about $10^{5} \mathrm{~K}$ and emits brightly at optical, ultraviolet, and X-ray wavelengths during the period from about 100 days to a few years. Komossa (2015) described observational appearances of these events in detail.

Swift 164449.3+573451 (hereafter Swift J1644+57) was initially discovered as a $\gamma$-ray burst (GRB) event. The Swift Burst Alert Telescope (BAT, 15-150 keV) detected a new uncataloged source on 2011 March 28. Because the source was assumed to be a GRB, it was named as GRB 110328A. However, this first detection was also followed by three additional flares with a subsequent increase of the count rate over the next two days and thus, it was recognized that this object was not a GRB. Now, the name of the source, Swift J1644+57 based on the initial position of its X-ray counterpart is commonly used.
Swift $\mathrm{J} 1644+57$ is also characterized by the long duration of the X-ray outburst (with a power-law decay $\propto t^{-5 / 2}$ ) and specific flaring events. We also note that the spatial coincidence of it with the central nucleus of a galaxy at redshift 0.354 (the luminosity distance, $1.5 \mathrm{Gpc}$, see Levan et al. 2011) indicates that this source was not a GRB, but associated with an accretion event in the close vicinity of a SMBH (see also Bloom et al. 2011; Burrows et al. 2011).

We should emphasize that this TDE phenomenon is only occasionally observed and therefore is poorly studied. Furthermore, there are no direct measurements of the mass, $M_{\mathrm{BH}}$ of a dormant BH except of our Galactic center black hole. In the Swift J1644+57 case the optical emission lines imply that the host object is not an active galactic nucleus (AGN), but a HIItype galaxy. Variable emission was also detected in the nearIR and was not detected in the optical band (possibly due to the excess of extinction in the optical spectrum). Various estimates of a BH mass, based on the rapid variability timescale, all give that $10^{7}<M_{\mathrm{BH}}<10^{9} M_{\odot}$. This indirect constraints provide only an upper limit to the $\mathrm{BH}$ mass (see R88), for example, a minimum variability time of $\sim 100 \mathrm{~s}$ that sets an upper limit to the light-crossing time (Bloom et al. 2011; Burrows et al. 2011; Liang \& Liu 2003) and well-known empirical relations between $M_{\mathrm{BH}}$ and the host galaxy environment (Silk \& Rees 1998; Jahnke \& Maccio 2011). Based on optical luminosity of the 
host galaxy, Levan et al. (2011) evaluated a spheroidal mass, $M_{\text {sph }} \sim 10^{9}-10^{10} M_{\odot}$. Using the log-linear spheroidal mass and the BH mass relation of Bennert et al. (2011), Levan et al. found that likely a BH mass within $2 \times 10^{6} \leq M_{\mathrm{BH}} \leq 10^{7} M_{\odot}$ taking into account the relation by Graham (2012) instead of the relation, $M_{\mathrm{sph}}-M_{\mathrm{BH}}$. The latter relation also provides a lower range of $\mathrm{BH}$ masses, $10^{5} M_{\odot} \leq M_{\mathrm{BH}} \leq 10^{7} M_{\odot}$ which is more consistent with the BH mass, $\log \left(M_{\mathrm{BH}} / M_{\odot}\right)=5.5 \pm 1.1$ estimated by Miller \& Gultekin (2011), based on empirical, so called fundamental plane relations between radio and X-ray luminosities of accreting BHs. Many subsequent observations of Swift J1644+57 have been performed at all wavelengths (X-rays: Mangano et al. 2016; Gonzalez-Rodriguez 2014; Castro-Tirado et al. 2013; Zauderer et al. 2013; Saxton et al. 2012; Reis et al. 2012; radio: Cendes et al. 2014; Zauderer et al. 2013; Berger et al. 2012; IR and radio polarimetry: Wiersema et al. 2012). Aliu et al. (2011). Aleksic et al. (2013) reported non-detections of Swift J1644+57 using MAGIC and VERITAS for energies greater $100 \mathrm{GeV}$. Evidence for quasi-periodical oscillations (QPOs) in the X-ray power spectrum of Swift J1644+57 with a period of $\sim 200 \mathrm{~s}$ in X-rays was suggested by Reis et al. (2012) and Saxton et al. (2012). In contrast to the long-term X-ray lightcurve, the radio emission of Swift J1644+57 continued to rise (Zauderer et al. 2013; Berger et al. 2012).

The Swift X-ray lightcurve of Swift J1644+57 decreased and then (at 507th day from the discovery) showed a sudden drop by a factor of $\sim 200$, that is, it was no longer detectable with Swift but only detectable by Chandra (Zauderer et al. 2013) and $X M M-N e w t o n$ in longer time pointings (Gonzalez-Rodriguez et al. 2014). A second event very similar to Swift J1644+57 was discovered by the Swift/BAT on May 27, 2011 (Krimm et al. 2011) from Swift J2058+0516, hereafter Swift J2058+05. This TDE source is located at a higher redshift, 1.186 (Komossa 2015; Cenko et al. 2012).

The evidence for a possible TDE in Swift J2058+05 was reported by Cenko et al. (2012) who showed that this source demonstrated a luminous, long-term $\mathrm{X}$-ray outburst with a peak luminosity of $L_{\mathrm{x}} \sim 3 \times 10^{47} \mathrm{erg} / \mathrm{s}$. The event was also accompanied by a strong radio emission (Pasham et al. 2015). The associated host galaxy of Swift J2058+05 was optically inactive. Because of the many similarities between Swift 2058+05 and Swift J1644+57, Cenko et al. (2012) suggested that a similar outburst mechanism is consistent with multi-wavelength followup observations (Pasham et al. 2015). The X-ray lightcurve of Swift J2058+05 is similar to that of Swift J1644+57 which shows an abrupt drop after 250-300 days since the initial outburst. Because of rapid variability of the X-rays before the drop, an origin was associated with the vicinity of SMBH rather than with the forward shock location (Pasham et al. 2015).

The BH mass limits of Swift J2058+05 were derived based on the X-ray turnoff $\left(10^{4} M_{\odot} \leq M_{\mathrm{BH}} \leq 2 \times 10^{6} M_{\odot}\right.$, as well as using the $\mathrm{BH}$ mass upper limit estimate method of applying the X-ray variability timescale, $5 \times 10^{7} M_{\odot}$ (see Pasham et al. 2015). Furthermore, Pasham et al. (2015) assumed that the TDE source optical flux is dominated by the host galaxy and they constrained the BH mass of the central SMBH using the well-known bulge luminosity as a function of BH mass relations (e.g., Lauer et al. 2007). As a result they inferred the $\mathrm{SMBH}$ mass of about $M_{\mathrm{BH}} \leq$ $3 \times 10^{7} M_{\odot}$.

It is desirable to have an independent $\mathrm{BH}$ identification for its central object as well as the $\mathrm{BH}$ mass determination by an alternative to the aforementioned methods, based on luminosity and minimal variability time estimates only. A method of the $\mathrm{BH}$ mass determination was developed by
Shaposhnikov \& Titarchuk (2009, hereafter ST09), using a correlation scaling between X-ray spectral and timing (or mass accretion rate) properties observed for many Galactic $\mathrm{BH}$ binaries during their spectral state transitions.

The origin of the X-ray emission in TDE is still unclear. However, this is for Swift J1644+57, there are two, well detected, prominent peaks in the spectral energy distribution: one in the far-infrared and another in the hard X-ray band (Bloom et al. 2011; Burrows et al. 2011). They can be modeled as a direct synchrotron emission (single-component model) from radio to X-rays, with strong dust extinction in the optical and ultraviolet bands. Alternatively, the radio and IR peaks are related to the synchrotron emission and the X-ray peak is due to inverse Compton scattering of external photons, most likely disk photons (two-component blazar model). Another possibility is that the X-ray emission is due to the inverse Compton emission at the base of the jet, while radio and IR synchrotron emission comes from the forward shock at the interface between the head of the jet and the interstellar medium (Bloom et al. 2011).

The goal of the present study is to investigate the peak in X-ray observational data from Swift J1644+57 and Swift J2058+05 and to infer their fundamental observational characteristics. In particular, the soft X-ray spectrum of Swift J1644+57 was fitted by a simple absorbed power law, although more complex spectral models were also discussed (Burrows et al. 2011; Saxton et al. 2012). An average photon index during the first year was $\Gamma \sim 1.8$ (Burrows et al. 2011; Levan et al. 2011). However, the physical meaning of this average value has to be interpreted more carefully, as there were strong variations in hardness and in the photon index between flares, with a change between $\Gamma \approx 1.3$ and 3 (Levan et al. 2011). In particular, the photon index was harder when the source was brighter (Burrows et al. 2011; Kennea et al. 2011; Levan et al. 2011).

Our main goal in the present study is the full analysis of the Swift/XRT follow-up data of Swift J1644+57 from the beginning to the end of the 2011-2012 outburst and Swift J2058+05 during 2011 outburst decay. We present spectral analysis and interpretation of RXTE, Swift and Suzaku data of the sources. In Sect. 2 we show the list of observations used in our analysis, while in Sect. 3 we provide details of X-ray spectral fittings. We discuss an evolution of the X-ray spectral properties during the high-low state transitions in Sect. 4 and demonstrate the results of the scaling analysis, in order to estimate BH masses of Swift J1644+57 and Swift J2058+05 in Sect. 5. We make our conclusions in Sect. 6.

\section{Observations and data reduction}

Swift J1644+57 was observed by Suzaku during April 6, 2011 and May 17, 2012 (see Sect. 2.1) along with the short-term RXTE observations on March 30-31, 2011 which we describe in Sect. 2.2 and by Swift during the period of 2011-2016 (see Sect. 2.3). The RXTE data (three observations) are related to the peak burst phase and probe harder X-ray energies (3-100 keV). In contrast, the well-exposed Suzaku data are very advantageous in determining low-energy photoelectric absorption, which is presumably not associated with the source directly.

Short-term RXTE observations of of Swift J2058+05 on June 1, 2011 are described in Sect. 2.2. We also used publicly available data by the Swift Observatory obtained from May 27, 2011 to December 7, 2011 (see Sect. 2.3). We extracted these data from the HEASARC archives and found that the Swift data cover the decay phase of X-ray outburst for Swift J1644+57 and Swift 2058+05, as well as partly, catch the peak burst interval. 
Table 1. List of the Suzaku observations of Swift J1644+57 in the $0.3-10 \mathrm{keV}$ range used in our analysis.

\begin{tabular}{lllllc}
\hline \hline Number of set & Obs. ID & Start time (UT) & End time (UT) & MJD interval & $\begin{array}{c}\text { Mean count rate } \\
\text { (cts/s) }\end{array}$ \\
\hline Sz1 ............... & $906001010^{1}$ & 2011 April 6 02:24:43 & 2011 April 7 00:32:17 & $55657.1-55658.0$ & $4.59 \pm 0.03$ \\
Sz2 .............. & $707018010^{1}$ & 2012 May 17 17:30:38 & 2012 May 19 01:15:11 & $56064.7-56066.1$ & $0.032 \pm 0.001$ \\
\hline
\end{tabular}

References. ${ }^{(1)}$ Usui \& Kawai (2015).

Table 2. List of the RXTE observations of Swift J1644+57 and Swift J2058+05.

\begin{tabular}{llllll}
\hline \hline Source & $\begin{array}{l}\text { Number } \\
\text { of set }\end{array}$ & Obs. ID & Start time (UT) & End time (UT) & MJD interval \\
\hline Sw J1644+57 & R1 ......... & $96424-01-01-00$ & 2011 March 30 04:53:04 & 2011 March 30 05:26:56 & $55650.20-55650.27$ \\
& R2 .......... & $96424-01-01-01$ & 2011 March 31 02:51:12 & 2011 March 31 04:09:36 & $55651.11-55651.17$ \\
& R3 ......... & $96424-01-01-02$ & 2011 March 31 04:39:12 & 2011 March 31 04:57:04 & $55651.19-55651.21$ \\
\hline Sw J2058+05 & R4 ......... & $96431-01-01-00$ & 2011 June 1 01:45:36 & 2011 June 1 02:12:00 & 55713.07-55 713.09 \\
\hline
\end{tabular}

A summary of the X-ray observations analyzed in this work is given in Tables $1-3$.

\subsection{Suzaku}

We studied the TDE source, Swift J1644+57 using the Suzaku data, for April 6 of 2011 and May 17 of 2012 observations (see Table 1). These particular observations correspond to nine and 416 days after the source discovery. We used HEASOFT software package (version 6.13) and calibration database (CALDB) released on February 10, 2012 for XIS. Since background is dominant in the lower energy band, we used photons in the 1-10 keV (for XIS0, and 3) and 1-7 keV (for XIS 1) energy bands.

The data reduction and spectral analysis are performed following the Suzaku Data Reduction Guide ${ }^{1}$. We obtained cleaned event files by re-running the Suzaku pipeline implementing the latest calibration database (CALDB) available since January 20 of 2013, and also applying the associated screening criteria files.

We extracted spectra from the cleaned event files using XSELEC and we generated responses for each detector utilizing the XISRESP script with a medium resolution. The spectral and response files for the front-illuminated detectors (XIS0, 1 and 3) were combined using the FTOOL ADDASCASPEC, after confirmation of their consistency. In addition, we grouped the spectra in order to have a minimum of 20 counts per energy bin.

We carried out spectral fitting applying XSPEC v12.7.1. The energy ranges around of 1.75 and $2.23 \mathrm{keV}$ are not used for spectral fitting because of the known artificial structures in the XIS spectra around the Si and Au edges. Therefore, for spectral fits we chose the $0.3-10 \mathrm{keV}$ range for the XISs (excluding 1.75 and $2.23 \mathrm{keV}$ points).

\subsection{RXTE}

We analyzed three RXTE observations of Swift J1644+57 made between July 1997 and January 2001 related to different spectral states of the source. In addition, we analyzed only one RXTE observation of Swift J2058+05 made on June 1, 2011 close to peak burst state of the source.

\footnotetext{
1 http://heasarc.gsfc.nasa.gov/docs/suzaku/analysis/
}

Standard tasks of the LHEASOFT/FTOOLS 5.3 software package were applied for data processing. For spectral analysis we used PCA Standard 2 mode data, collected in the 3$23 \mathrm{keV}$ energy range, using PCA response calibration (ftool pcarmf v11.7). The standard dead time correction procedure was applied to the data. In order to construct the broadband spectra of the data we also used HEXTE detectors in the case of Swift J1644+57. The spectral analysis of the data in the 19$150 \mathrm{keV}$ energy range was implemented in order to account for the uncertainties in the HEXTE response and background determination. We subtracted a background corrected in off-source observations. The data are available through the GSFC public archive $^{2}$. Systematic error of $0.5 \%$ was applied to all analyzed RXTE spectra.

In Table 2 we listed the groups of RXTE observations of Swift J1644+57 and Swift J2058+05 tracing thoroughly the source evolution during different spectral states. We modeled the RXTE energy spectra using XSPEC astrophysical fitting software.

\subsection{Swift}

The observational set of Swift J1644+57 is extensive. The source was monitored by the XRT several times a day, every day since March 28, 2011. We used the Swift observations carried out from 2011 to 2016. The log of the Swift/XRT observations used in this paper is shown in Table 3 . We analyzed only photon-counting mode (PC) data. The Swift-XRT/PC data (ObsIDs, shown in the second column of Table 3) were processed using the HEASOFT v6.14, the tool XRTPIPELINE v0.12.84 and the calibration files (CALDB version 4.1). The ancillary response files were created using XRTMKARF v0.6.0 and exposure maps generated by XRTEXPOMAP v0.2.7. We fitted the spectrum using the response file SWXPC0TO12S6_20010101v012.RMF. We also used the online XRT data product generator ${ }^{3}$ for independent check: light curves and spectra, including background and ancillary response files (see Evans et al. 2007, 2009).

The log of the Swift/XRT observations of Swift J2058+05 used in this paper is also shown in Table 3. The Swift-XRT/PC data (ObsIDs) are indicated in the third column of this table

\footnotetext{
2 http://heasarc.gsfc.nasa.gov

3 http://wWw.swift.ac.uk/user_objects/
} 
Table 3. List of the Swift observations of Swift J1644+57 and Swift J2058+05.

\begin{tabular}{|c|c|c|c|c|c|}
\hline Source & $\begin{array}{l}\text { Number } \\
\text { of set }\end{array}$ & Obs. ID & Start time (UT) & End time (UT) & MJD interval \\
\hline \multirow[t]{5}{*}{ Sw J1644 } & Sw1 .................... & $\begin{array}{l}00031955(002-106,107-177,179-181, \\
183-211,213-255^{1,2,3,4,5,6}\end{array}$ & 2011 Mar. 31 & 2011 Dec. 6 & $55651.0-55901.0$ \\
\hline & ................ & $\begin{array}{l}00032200(001-210,212-217,219 \\
221-225,227-232,234-237^{1,2,3}\end{array}$ & 2011 Dec. 7 & 2012 Aug. 15 & $55902.3-56154.1$ \\
\hline & Sw3 …............... & $\begin{array}{l}00032526(001-003,005-110,103-116 \\
118-255^{2,3}\end{array}$ & 2012 Aug. 16 & 2015 Apr. 24 & $56155.9-57136.2$ \\
\hline & Sw4 .................. & $00033765(001-005,007-060,062-079)$ & 2015 May 1 & 2016 Oct. 21 & $57143-57682$ \\
\hline & Sw5 .................. & $00450158(000-001,004-007)^{1,3,6}$ & 2011 Mar. 28 & 2011 Mar. 30 & $55648.6-55650.6$ \\
\hline \multirow[t]{2}{*}{ Sw J2058 } & Sw6. & $00032004001^{7,9}$ & 2011 May 27 & 2011 May 30 & $55708.9-55709.4$ \\
\hline & Sw7 ................. & $000320260(03-07,09-16,18-21)^{7,8,9}$ & 2011 July 11 & 2011 Dec. 7 & $55753.5-55902.3$ \\
\hline
\end{tabular}

References. (1) Saxton et al. (2012); (2) Komossa et al. (2015); (3) Mangano et al. (2016); (4) Burrows et al. (2011); (5) Levan et al. (2011); (6) Zauderer et al. (2013); (7) Pasham et al. (2015); (8) Komossa et al. (2015); (9) Cenko et al. (2012).

and they were processed using the same software as that in the case of Swift J1644+57. We also obtained images using the online XRT data processing facility ${ }^{4}$. In this way, we inspected the image in vicinity of Swift $2058+05$ to exlude the presence of other sources close to this source. For the Swift/XRT image $0.3-10 \mathrm{keV}$ of the source field of view (FOV) we use the circle of 5 arcmin radius with the center of the source position $(\alpha=$ $20^{\mathrm{h}} 58^{\mathrm{m}} 19.76^{\mathrm{s}}, \delta=+05^{\circ} 13^{\prime} 29.8^{\prime \prime}$, J2000.0; Cenko et al. 2012). The faint source is at the bottom of the image (at 5.8 arcmin radius), but it is beyond the FOV used for our analysis.

\section{Results}

\subsection{X-ray light curves}

The $0.3-10 \mathrm{keV}$ light curve of Swift J1644+57 is shown in Fig. 1 (top panel), for the time period 2011-2012. The source signal (with $2 \sigma$ detection level) for PC/WT mode is indicated, as is the background level for PC/WT mode, respectively. We found a complex count rate behavior at the burst peak, which is variable from 0.3 to 100 counts/s (in PC mode) within $100 \mathrm{~s}$, and a long slow decay (from 1 to 0.1 counts/s) with rough duration of 500 days. For the rest of the Swift observations the source remained in the low state. Specifically, on 508th day (from the first detection on March 28, 2011) the source emission suddenly dropped below the Swift detection limit (Sbarufatti et al. 2012). The light curve of Swift J2058+05 in the 0.3-10 keV range using Swift/XRT during the source outburs decay on from May 27 to July 11, 2011 is presented in Fig. 2.

\subsection{Hardness-intensity diagrams and HR curves}

In application to the Swift data of Swift J1644+57 we have defined the hardness ratio (HR) as a ratio of the hard and soft counts in the $1.5-10 \mathrm{keV}$ and $0.3-1.5 \mathrm{keV}$ bands, respectively. The HR value strongly depends on calculation of the background counts.

In the bottom panel of Fig. 1 we show the HR curve versus MJD time using PC mode data of Swift J1644+57. At the beginning, this curve shows variations tracking the light curve local flares, for which the hardness rises when the average rate increases, and then saturation when the average rate decreases.

\footnotetext{
4 See http://www.swift.ac.uk/user_objects/and Evans et al. (2007, 2009).
}

We observe a hardness plateau phase from $\sim 2.5$ to 16 days after the source discovery.

It is interesting that we detect a sharp hardening of X-ray emission flux of Swift J1644+57 near the initial outburst part, which is typical for known Galactic BHs (GBHs) and usually accompanied by the jet turn-on (e.g., in GRS 1915+105, see Belloni \& Motta 2016; Migliari \& Fender 2006; Titarchuk \& Seifina 2009).

In Fig. 3 we present the hardness-intensity diagram (HID), which demonstrates that different count-rate observations are assocated with different color regimes. The HR larger values correspond to harder spectra. A Bayesian approach was used to estimate the HR values and their errors (see Park et al. 2006) ${ }^{5}$. For clarity, we plot only one point with error bars (in the bottom right corner) to demonstrate typical uncertainties for the count rate and $\mathrm{HR}$.

Figure 3 clearly shows that the HR monotonically drops with the soft count rate $(0.3-1.5 \mathrm{keV})$. This particular sample is similar to those of most of outbursts of Galactic X-ray binary transients (see Homan et al. 2001; Belloni et al. 2006; Shaposhnikov \& Titarchuk 2006; ST09; TS09; Shrader et al. 2010; MunozDarias et al. 2014).

\subsection{X-ray spectral analysis}

A number of spectral models was used in order to test them for all available data sets for Swift J1644+57 and Swift J2058+05. Our goal is to establish an evolution between the low hard and high soft states based on spectral modeling.

\subsubsection{Swift J1644+57}

The X-ray spectra of Swift J1644+57 obtained using the Suzaku/XIS observations in 2011 (the high soft state) and 2012 (the low hard state. In Fig. 4 we show examples of these spectra along with that obtained by the Swift/XRT. Two $E F_{\mathrm{E}} S u z a k u$ spectral diagrams related to the high soft state and the low hard state are presented in Fig. 5. While the spectrum detected by RXTE and the combined Swift-RXTE spectral diagram are shown in Figs. 6 and 7, respectively.

\footnotetext{
5 A Fortran and C-based program which calculates the ratios using the methods described by Park et al. (2006; see http://hea-www . harvard.edu/AstroStat/BEHR/).
} 
E. Seifina et al.: Swift J1644+57 and Swift J2058+05. Black hole mass estimates
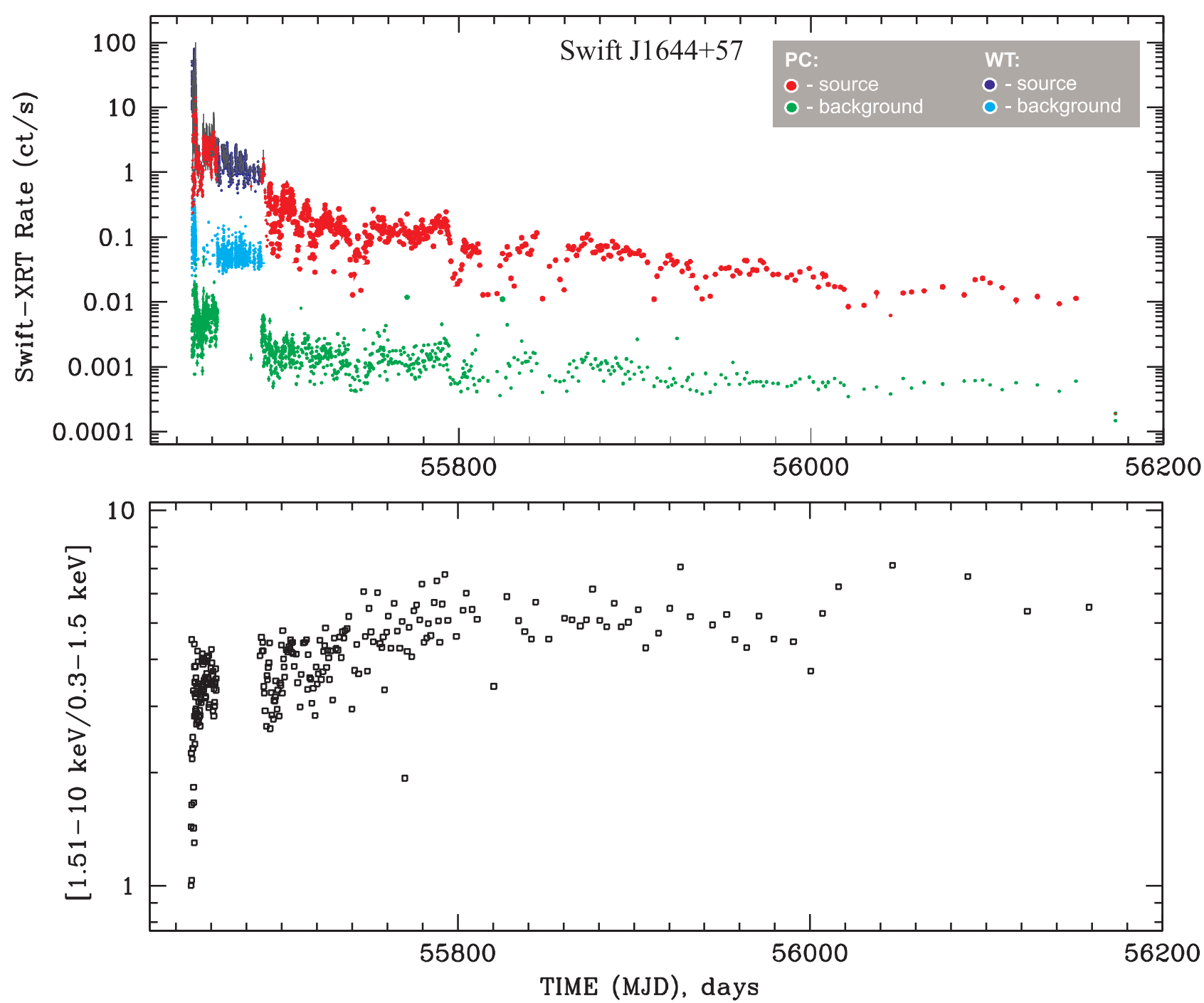

Fig. 1. Swift/XRT light curve of Swift J1644+57 in the 0.3-10 keV range during 2011-2012 (top panel). Here, red, blue points mark the source signal (with $2 \sigma$ detection level) for PC/WT mode. Green and cyan points indicate the background level for PC/WT mode, respectively. In the bottom panel we show the hardness ratio curve (1.51-10 keV/0.3-1.5 keV) using PC mode data of Swift J1644+57.

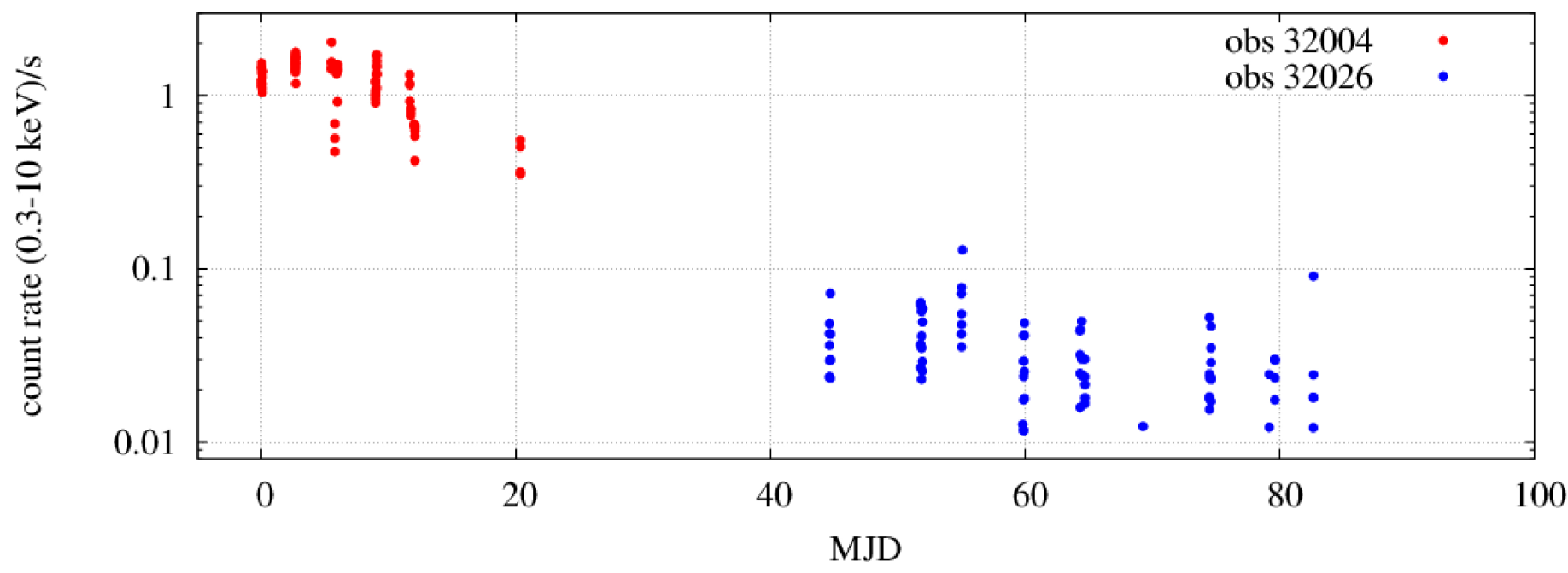

Fig. 2. Light curve of Swift J2058+05 in the 0.3-10 keV energy range during the outburst decay (2011, May, 27-July, 11) deteted by Swift/XRT. The red points mark the brighter phase $(\mathrm{id}=32004)$ of outburst, while the blue points indicate the fainter phase $(\mathrm{id}=32026)$. In the horizontal axis we show the time scale in the MJD-55 708.9 units 
Swift/XRT

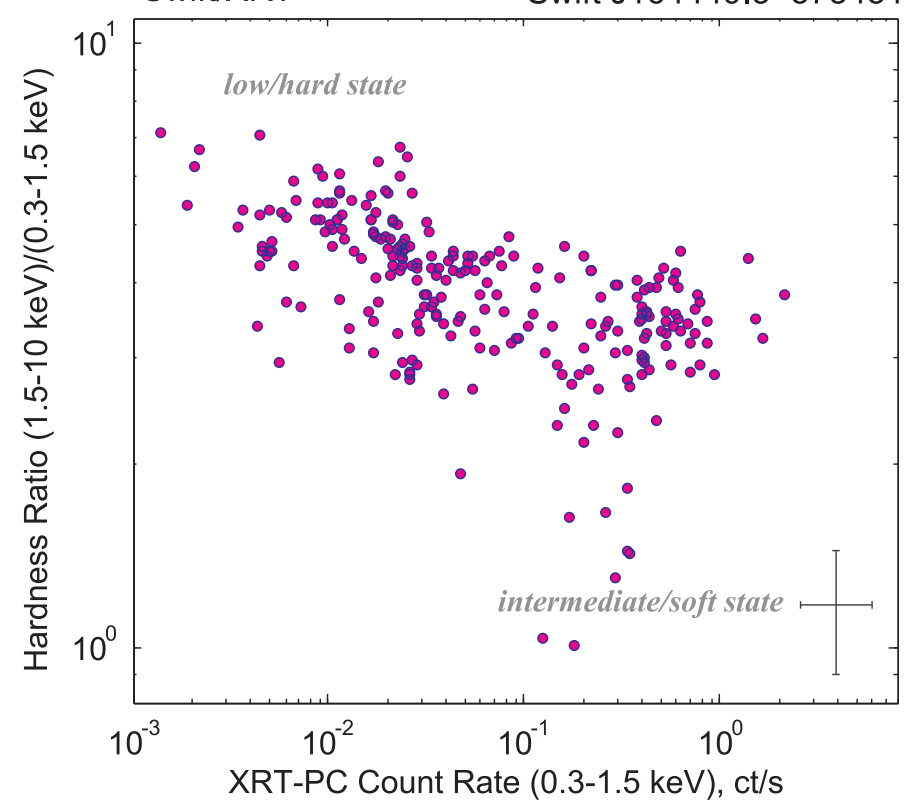

Fig. 3. Hardness-intensity diagram (HID) for Swift J1644+57 using the Swift observations (2011-2012) during spectral evolution from the high state to the low state. In the vertical axis, the hardness ratio (HR) is a ratio of the source counts in the two energy bands: the hard (1.5$10 \mathrm{keV}$ ) and soft (0.3-1.5 keV). HR decreases with a source brightness in the $0.3-10 \mathrm{keV}$ range (horizontal axis). For clarity, we plot only one point with error bars (in the bottom right corner) to demonstrate typical uncertainties for the count rate and HR.

We investigated the Suzaku spectra for Swift J1644+57 to check the following XSPEC spectral models: powerlaw, blackbody, the bulk motion Comptonization (BMC) and their possible combinations modified by an absorption model. We find that the absorbed power-law model (phabs $*$ zphabs $* z$ powerlw) reveals a statistically-unacceptable fits with the photon index $\Gamma_{\text {pow }} \sim 1.9$ and the reduced chi-squared $>2(S z 1$, see Tables 1,4$)$. A similar result was also previously reported by Burrows et al. (2011) and Reis et al. (2012). Moreover, the thermal model (zbbody) gives us even worse fits. As a result we checked a combination of $z$ bbody and zpowerlw models. In this case the model parameters are $N_{\mathrm{H}, z=0.354}=(1.2-1.9) \times 10^{22} \mathrm{~cm}^{-2} ; k T_{z b b}=0.24-0.3 \mathrm{keV}$ and $\Gamma=1.3-1.9$ (see more detail in Table 4). The best fits of the Suzaku spectra has been found using the BMC XSPEC model (Titarchuk et al. 1997), for which $\Gamma$ ranges from 1.1 to 1.8 for all observations (see Table 4 and Figs. 4-5). In Fig. 4 we also show the simultaneous spectra of Swift J1644+57 obtained using the Suzaku and Swift/XRT observations.

Thus, we decided to analyze all available spectral data of Swift J1644+57 using the XSPEC BMC model. The BMC model uses a convolution of a seed blackbody with an upscattering Green's function, presented in the framework of the BMC as a broken power law in which left and right wings have the spectral indices $\alpha+3$ and $\alpha$, respectively (see a description of the BMC Comptonization Green's function in Titarchuk \& Zannias 1998, TZ98; and compare with Sunyaev \& Titarchuk 1980). The BMC model has as the main parameters, $\alpha, A$ (related to the illumination fraction $f=A /(1+A)$ ), the seed blackbody (BB) temperature $T_{\mathrm{s}}$, and the $\mathrm{BB}$ normalization which is proportional to the seed BB luminosity and inversely proportional to $d^{2}$ where $d$ is a distance to the source (see also Titarchuk \& Seifina 2016a, hereafter TS16a).
Table 4. Best-fit parameters of the Suzaku spectra of Swift $\mathrm{J} 1644+57$ in the $0.45-10 \mathrm{keV}$ range using the following four models: phabs*zphabs*powerlw, phabs*zphabs*zbbody, phabs*zphabs(zbbody+zpowerlw) and phabs*zphabs*bmc.

\begin{tabular}{llll}
\hline \hline & Parameter & 906001010 & 707018010 \\
\hline Model & & & \\
\hline phabs & $N_{\mathrm{H}, \mathrm{Gal}}$ & $0.02^{f}$ & $0.02^{f}$ \\
zphabs & $N_{\mathrm{H}, z=0.354}$ & $0.9 \pm 0.1$ & $0.8 \pm 0.1$ \\
zpowerlw & $\Gamma_{\text {zpow }}^{\dagger \dagger}$ & $1.93 \pm 0.01$ & $1.3 \pm 0.1$ \\
& $N_{\text {zpow }}^{\dagger \dagger}$ & $33.2 \pm 0.5$ & $0.11 \pm 0.03$ \\
\hline & $\chi^{2}$ (d.o.f.) & $2.12(1568)$ & $1.25(177)$ \\
\hline phabs & $N_{\mathrm{H}, \mathrm{Gal}}$ & $0.02^{f}$ & $0.02^{f}$ \\
zphabs & $N_{\mathrm{H}, z=0.354}$ & $0.21 \pm 0.04$ & $0.2 \pm 0.1$ \\
zbbody & $k T_{z b b}(\mathrm{keV})$ & $1.27 \pm 0.01$ & $1.9 \pm 0.1$ \\
& $N_{z b b}{ }^{\dagger \dagger}$ & $1.07 \pm 0.02$ & $0.009 \pm 0.003$ \\
\hline & $\chi^{2}$ (d.o.f.) & $5.07(1568)$ & $1.54(177)$ \\
\hline phabs & $N_{\mathrm{H}, \mathrm{Gal}}$ & $0.02^{f}$ & $0.02^{f}$ \\
zphabs & $N_{\mathrm{H}, z=0.354}$ & $1.24 \pm 0.03$ & $1.9 \pm 0.4$ \\
zbbody & $k T_{z b b}(\mathrm{keV})$ & $0.29 \pm 0.02$ & $0.24 \pm 0.03$ \\
& $N_{z b b}{ }^{\dagger \dagger}$ & $0.16 \pm 0.09$ & $0.03 \pm 0.01$ \\
zpowerlw & $\Gamma_{\text {pow }}^{\dagger}$ & $1.92 \pm 0.02$ & $1.3 \pm 0.1$ \\
& $N_{\text {pow }}^{\dagger \dagger}$ & $32.5 \pm 0.8$ & $0.01 \pm 0.07$ \\
\hline & $\chi^{2}$ (d.o.f.) & $1.03(1566)$ & $1.17(175)$ \\
\hline phabs & $N_{\mathrm{H}, \mathrm{Gal}}$ & $0.02^{f}$ & $0.02^{f}$ \\
zphabs & $N_{\mathrm{H}, z=0.354}$ & $1.56 \pm 0.01$ & $1.5 \pm 0.2$ \\
bmc ${ }^{z}$ & $\Gamma_{\mathrm{BMC}}$ & $1.76 \pm 0.01$ & $1.2 \pm 0.1$ \\
& $k T_{\mathrm{S}}(\mathrm{eV})$ & $189 \pm 3$ & $270 \pm 7$ \\
& $\log \mathrm{A}$ & $-0.7 \pm 0.2$ & $-0.3 \pm 0.2$ \\
& $N_{\text {bmc }}{ }^{\dagger \dagger}$ & $0.47 \pm 0.07$ & $0.0002 \pm 0.0001$ \\
\hline & $\chi^{2}$ (d.o.f.) & $0.98(1566)$ & $0.97(175)$ \\
\hline & & & \\
\hline
\end{tabular}

Notes. Errors are given at the $90 \%$ confidence level. The normalization parameters of blackbody and BMC components are in units of $L_{36}^{\text {soft }} / d_{10}^{2} \mathrm{erg} \mathrm{s}^{-1} \mathrm{kpc}^{-2}$, where $L_{36}^{\text {soft }}$ is the soft photon luminosity in units of $10^{36} \mathrm{erg} \mathrm{s}^{-1}, d_{10}$ is the distance to the source in units of $10 \mathrm{kpc}$, and power-law component is in units of $k T_{\mathrm{BB}}$ and $k T_{\mathrm{s}}$ are the temperatures of the blackbody and seed photon components (in $\mathrm{keV}$ and $\mathrm{eV}$ ), respectively. $\Gamma_{\text {pow }}$ and $\Gamma_{\mathrm{BMC}}$ are the indices of the zpowerlw and bmc, respectively. Hereafter superscript ${ }^{z}$ indicates that the cosmological reddening of the spectrum was talen into ccount $[E \rightarrow E(1+z)]$. Redshift $z$ was fixed to 0.354 (Levan et al. 2011). $N_{\mathrm{H}, \mathrm{Gal}}$ and $N_{\mathrm{H}, z=0.354}$ are in units of $10^{22} \mathrm{~cm}^{-2}$. ${ }^{(f)}$ Indicates that a parameter was fixed.

The TDE source, Swift $\mathrm{J} 1644+57$ is located at $z=0.354$ and thus, we should take into account the cosmological reddening of the spectrum due to the redshifted energy band $(E \rightarrow E(1+z))$. In order to make this redding corrections we used the energies XSPEC command, which extends the maximum energy over which the model is being calculated to $(1+z)$ times the maximum energy in the response. As a result the $\mathrm{BMC}^{z}$ model describes the outgoing spectrum as a convolution of the input seed blackbodylike spectrum, of which normalization is $N_{\mathrm{BMC}}$ and color temperature is $k T_{\mathrm{s}}$, with the Comptonization Green's function.

In addition to the Galactic absorption, $N_{\mathrm{H}, \mathrm{Gal}}=2 \times 10^{20} \mathrm{~cm}^{-2}$ in this sky direction we also apply a multiplicative zphabs model with a column density, $N_{\mathrm{H}, z=0,354}$ of $1.5 \times 10^{22} \mathrm{~cm}^{-2}$ in the local (comoving) frame at redshift of 0.354 . As a result we obtained the best-fit results using the same model for the Suzaku and Swift spectral data of Swift J1644+57 in the $0.3-10 \mathrm{keV}$ energy range 
Table 5. Best-fit parameters of the RXTE spectra of Swift J1644+57 and Swift J2058+05.

\begin{tabular}{|c|c|c|c|c|c|c|c|c|}
\hline Source & Number of set & $\alpha=\Gamma-1$ & $k T_{\mathrm{s}}(\mathrm{eV})$ & $\log (\mathrm{A})$ & $N^{a}$ & $E_{\text {cut }}(\mathrm{keV})$ & $k T_{\mathrm{e}}(\mathrm{keV})$ & $\chi_{\text {red }}^{2}$ (d.o.f.) \\
\hline \multirow[t]{4}{*}{ Sw J1644 } & $\mathrm{R} 1^{b}$ & $0.76 \pm 0.01$ & $90^{d}$ & $0.5 \pm 0.3$ & $1.1 \pm 0.1$ & $90 \pm 4$ & & $0.90(74)$ \\
\hline & $\mathrm{R} 2^{b}$. & $0.73 \pm 0.02$ & $90^{d}$ & $-0.26 \pm 0.08$ & $0.6 \pm 0.2$ & $100 \pm 7$ & & $0.95(74)$ \\
\hline & $\mathrm{R} 3^{b}$. & $0.78 \pm 0.04$ & $90^{d}$ & $-0.3 \pm 0.1$ & $0.5 \pm 0.1$ & $110 \pm 8$ & & $0.95(74)$ \\
\hline & $\mathrm{R} 1+\mathrm{Sz}^{c} \ldots \ldots$. & $0.79 \pm 0.05$ & $370 \pm 10$ & $-0.43 \pm 0.04$ & $0.4 \pm 0.1$ & $\ldots .$. & $34 \pm 2$ & 1.03 (899) \\
\hline \multirow[t]{2}{*}{ Sw J2058 } & $\mathrm{R} 4^{b} \ldots \ldots \ldots \ldots \ldots$ & $0.6 \pm 0.1$ & $160^{d}$ & $0.3 \pm 0.1$ & $3.4 \pm 0.5$ & $16 \pm 4$ & & $0.93(125)$ \\
\hline & $\mathrm{R} 4+\mathrm{Sw}^{c} \ldots \ldots$ & $0.45 \pm 0.06$ & $210 \pm 30$ & $-0.43 \pm 0.04$ & $3.8 \pm 0.3$ & $\ldots$. & $4.0 \pm 0.8$ & $1.02(1094)$ \\
\hline
\end{tabular}

Notes. Errors are given at the $90 \%$ confidence level. ${ }^{(a)}$ The normalization parameters of the BMC and COMPTB components are in units of $L_{36}^{\text {soft }} / d_{10}^{2} \mathrm{erg} \mathrm{s}^{-1} \mathrm{kpc}^{-2}$, where $L_{36}^{\text {soft }}$ is the soft photon luminosity in units of $10^{36} \mathrm{erg} \mathrm{s}^{-1}, d_{10}$ is the distance to the source in units of $10 \mathrm{kpc}$. $N_{\mathrm{H}, \mathrm{Gal}}$, the Galactic column density for the neutral absorber, was fixed to $2 \times 10^{20} \mathrm{~cm}^{-2}$ (for Sw J1644+57) and $6.5 \times 10^{20} \mathrm{~cm}^{-2}$ (for Sw J2058+05; Kalberla et al. 2005) and $N_{\mathrm{H} z=0.354} / N_{\mathrm{H} z=1.1853}$, the column density for the neutral absorber in the local frame at redshift $z=0.354 / z=1.1853$, was fixed to $1.5 \times 10^{22} \mathrm{~cm}^{-2}$ (for Sw J1644+57; Saxton et al. 2012) and $2.6 \times 10^{21} \mathrm{~cm}^{-2}$ (for Sw J2058+05; Cenko et al. 2012), respectively; $k T_{\mathrm{s}}$ is the seed photon temperature, $k T_{\mathrm{e}}$ is the electron temperature, and $E_{\text {cut }}$ is the cutoff energy. ${ }^{(b)}$ Spectrum was fitted by the phabs*zphabs*bmc ${ }^{2 *}{ }^{*}$ zhighect model; ${ }^{(c)}$ spectrum was fitted by the phabs*zphabs*CompTB ${ }^{z}$ model; ${ }^{(d)}$ indicates that a parameter was fixed.
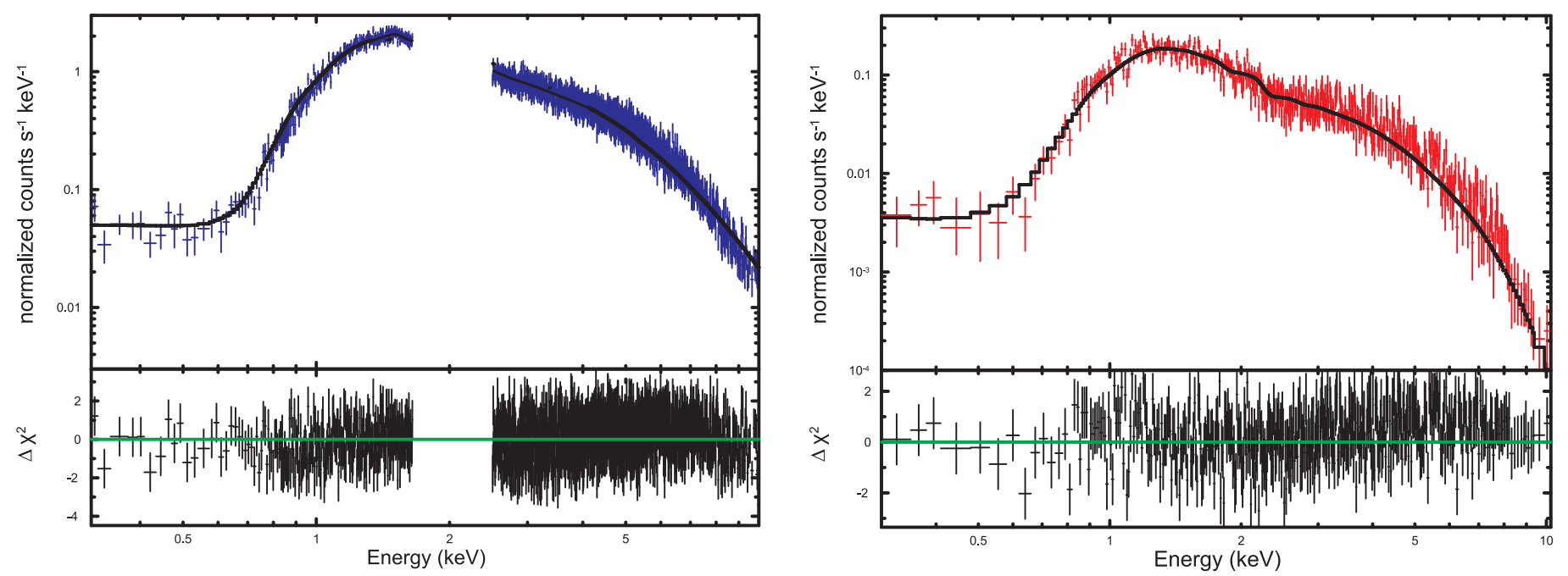

Fig. 4. Suzaku/XIS spectra (blue) and Swift/XRT (red) of Swift J1644+57 at the decay phase (both on 2011 April 6, MJD = 55 657) in normalized counts fitted using the phabs*zphabs*bmc ${ }^{z}$ model with $\alpha=0.63 \pm 0.01, \log (A)=-0.72 \pm 0.2, k T_{\mathrm{s}}=240 \pm 5 \mathrm{eV}$ for the Suzaku spectrum, $S z 1$ $\left(\chi_{\text {red }}^{2}=0.98\right.$ for 1566 d.o.f.) and with $\alpha=0.77 \pm 0.04, \log (A)=-1.62 \pm 0.09, k T_{\mathrm{s}}=200 \pm 20 \mathrm{eV}$ ( $\chi_{\text {red }}^{2}=1.04$ for 659 d.o.f.) for the Swift spectrum. The spectral models are shown by black hystograms.

throughout all (high soft and low hard) states. However, to fit the RXTE spectral data of Swift J1644+57 in a wide energy range we have to apply a multiplicative component zhighect to the $\mathrm{BMC}$ model in order to correctly reproduce the high energy part of the source spectrum (see Table 5 and Fig. 6). For the crosscheck, we combined Swift and RXTE spectra both obtained on March 31, 2011 and apply the Comptonization COMPTB model (see Fig. 7) which is the XSPEC model ${ }^{6}$, see Farinelli et al. (2008), for the direct determination of the high energy cutoff of the spectrum, $E_{\text {cut }}$ which is related to the plasma temperature $k T_{\mathrm{e}}$ for the thermal Comptonization $\left(E_{\text {cut }} \sim 2 k T_{\mathrm{e}}\right)$. The fit parameters of these models are shown in Table 5.

Using the same model, we made the spectral analysis of Suzaku, Swift and RXTE observations and found that Swift J1644+57 was in the three spectral states (the low hard, intermediate and soft states). The best-fit $\Gamma$ are presented in Tables 4, 5 (see also Figs. 4, 5). Evolution of the source between the low state and high state is accompanied by a monotonic increase of the normalization parameter, $N_{\mathrm{BMC}}$ from 0.5

\footnotetext{
6 http://heasarc.gsfc.nasa.gov/docs/software/lheasoft/ xanadu/xspec/models/comptb.html
}

to $300 \times L_{33} / d_{10}^{2} \mathrm{erg} / \mathrm{s} / \mathrm{kpc}^{2}$ which correlates with an increase of $\Gamma$ from 1.1 to 1.8 (see Fig. 8). It is also interesting that this source demonstrates monotonical growth of the radio flux density $(15.4 \mathrm{GHz})$ along with the drop of X-ray brightness (see second panel from the top in Fig. 9).

The rapid decline during 2011-2012 outburst decay is not seen in the radio implying that X-ray and radio emission at least have different origins at later times. In fact, Zauderer et al. (2013) interpreted the sudden drop in X-ray emission as evidence for a change in accretion regime. Specifically, Zauderer et al. related turning off the jet production, with the X-ray fainter stage. They also argued that the continued radio emission emananting from the forward shock related to the jet.

\subsubsection{Swift J2058+05}

For Swift J2058+05 we also investigated the Swift and RXTE spectra and checked the XSPEC spectral models which were powerlaw, blackbody, the BMC, and their possible combinations modified by an absorption model and redshift correction. 


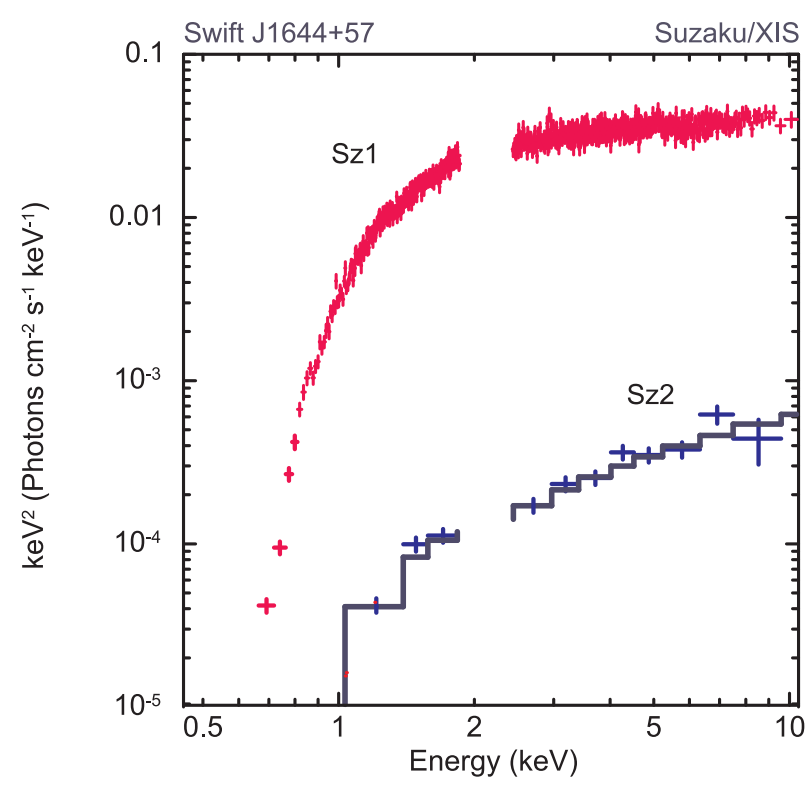

Fig. 5. Two $E F_{E}$ spectral diagrams during the high soft (purple) and low hard (blue) spectral states of Swift J1644+57. Data taken from Suzaku observations, 906001010 (Sz1, high soft and 707018010 (Sz2, low hard.

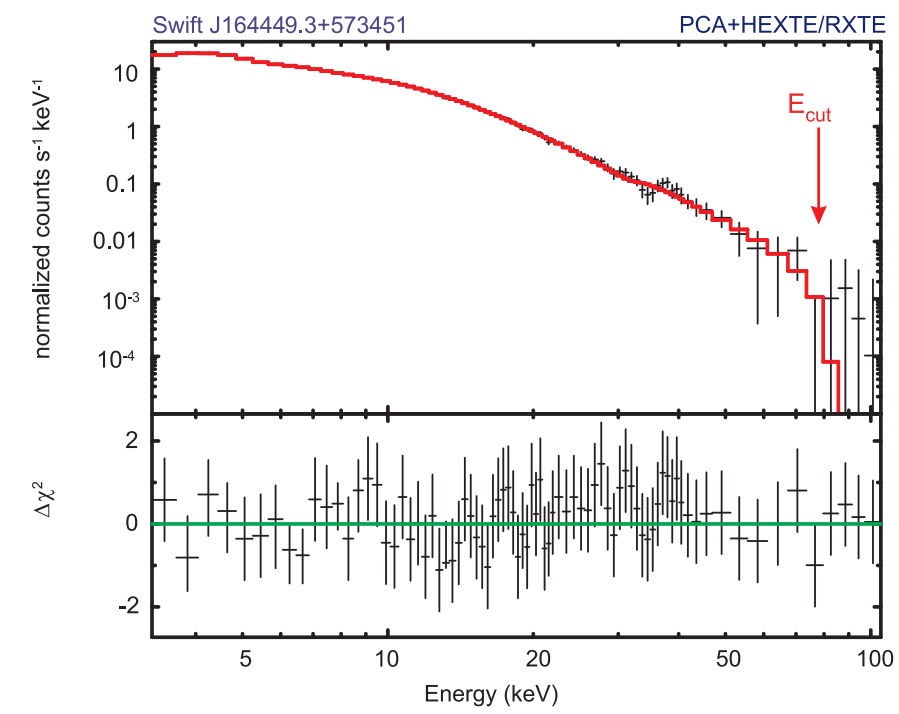

Fig. 6. RXTE (3-100) keV spectrum of Swift J1644+57. The observation taken on 2011 March $30(\mathrm{MJD}=55650.0, \mathrm{ID}=96242-01-01-00)$ was fitted using CompTB model with $\alpha=0.86 \pm 0.03, k T_{\mathrm{e}}=46 \pm 3 \mathrm{keV}$, $\log (A)=-0.5 \pm 0.2, k T_{\mathrm{s}}=0.3 \mathrm{keV}$ (fixed) with the intrinsic column density of $N_{\mathrm{H}, z=0.354}=1.5 \times 10^{22} \mathrm{~cm}^{-2}$ (at redshift $z=0.354$ ) and the Galactic absorption $N_{\mathrm{H}, \mathrm{Gal}}=2 \times 10^{20} \mathrm{~cm}^{-2}\left(\chi_{\text {red }}^{2}=0.97\right.$ for 120 d.o.f.). The high energy cut-off is clearly seen at $E_{\text {cut }}=80 \mathrm{keV}$.

We show examples of the Swift/XRT spectra in $E F_{E}$ and normalized count units (see Figs. 10, 11). In particular, Fig. 10 shows the best-fit spectrum during the intermediate-low hard state (IS-LHS) decay transition in $E F_{E}$ units (top panel) and $\Delta \chi$ (bottom panel). Figure 11 demonstrates the spectral evolution using three representative Swift/XRT spectra in normalized count units (top panel) with $\Delta \chi$ (bottom panel) for the LHS, the IS and the high soft state (HSS).

We also found, as that for Swift J1644+57, the absorbed power-law model (phabs * zphabs * zpowerlw) reveals a statistically-unacceptable fits (see Table 6 for id $=$ 00032004001). A similar result was also previously reported

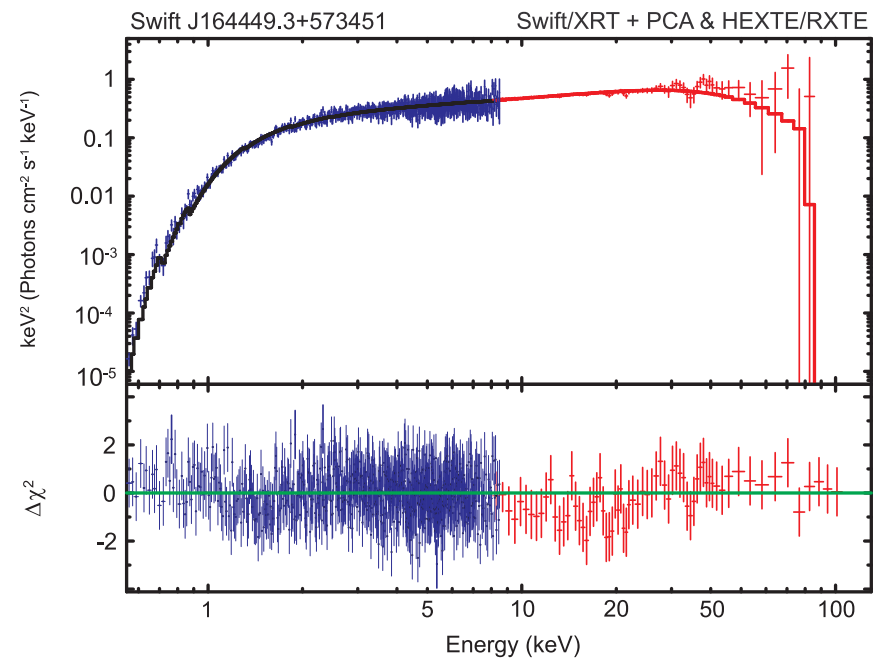

Fig. 7. Combined Swift/XRT and RXTE/PCA+HEXTE spectrum at the peak outburst phase (on 2011 March 31) in $E F(E)$ units fitted using phabs $*$ CompTB model (with $\alpha=0.79 \pm 0.05, k T_{\mathrm{e}}=34 \pm 2 \mathrm{keV}, \log (A)=$ $-0.43 \pm 0.04, k T_{\mathrm{s}}=370 \pm 10 \mathrm{eV}$ ( $\chi_{\text {red }}^{2}=1.03$ for 889 d.o.f. $)$, see also Table 5.

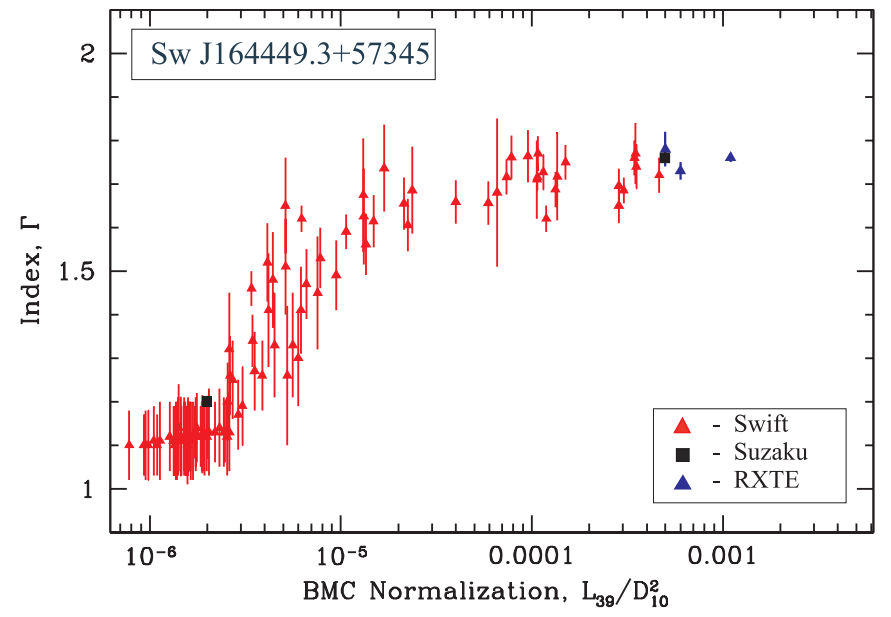

Fig. 8. Photon index, $\Gamma$ plotted versus BMC normalization (which is proportional to $\dot{M}$ ) for the TDE source, Swift J1644+57 using Swift (red triangles), Suzaku (black squares) and RXTE (blue triangles) data (see Tables 4 and 5).

by Cenko et al. (2012) and Pasham et al. (2015). Moreover, the thermal model (zbbody) gives us even worse fits.

We then checked a combination of zbbody and zpowerlw models. In this case the model parameters are $N_{\mathrm{H}, z=1.185}=$ $(1.9-2.3) \times 10^{21} \mathrm{~cm}^{-2} ; k T_{z b b}=1.2-5.7 \mathrm{keV}$ and $\Gamma=1.3-1.9$ (see more details in Table 6). However, the best fits of the Swift spectra has been found using the BMC XSPEC model (Titarchuk et al. 1997), for which $\Gamma$ also ranges from 1.2 to 1.8 for all observations similar to that for Swift J1644+57 (see Fig. 8). Thus, we decided to analyze all available spectral data of Swift J2058+05 using the XSPEC BMC model.

The TDE source, Swift J2058+05 is located at $z=1.185$ and thus, we should also take into account the cosmological reddening of the spectrum due to the redshifted energy band $(E \rightarrow E(1+z))$, see details in Sect. 3.3.1. We used the $\mathrm{BMC}^{z}$ model to describe the outgoing spectrum.

In addition to the Galactic absorption, $N_{\mathrm{H}, \mathrm{Gal}}=6.5 \times$ $10^{20} \mathrm{~cm}^{-2}$ in this sky direction we also apply a multiplicative zphabs model with a column density, $N_{\mathrm{H}, z=1.185}$ of $2.5 \times$ $10^{21} \mathrm{~cm}^{-2}$ in the local (comoving) frame at redshift 1.185 . We 
obtained the best-fit results using the same model for the Swift and RXTE spectral data of Swift J2058 in the 0.3-10 keV and 2$30 \mathrm{keV}$ energy ranges, respectively, throughout all spectral states (HSS, IS and LHS). However, to fit the RXTE spectral data of Swift $\mathbf{J} 2058+05$ in a wide energy range we should also apply (as for the case of Swift J1644+57) a multiplicative component zhighect to the BMC model in order to correctly reproduce the high energy part of the source spectrum (see Table 5 (lower part) and Fig. 7, related to the case of Swift J1644+57). For the crosscheck, as we did for the Swift J1644+57 data, we combined Swift and RXTE spectra, both obtained at the end of May, 2011, and apply the COMPTB model for the determination of $E_{\text {cut }}$ which is $E_{\text {cut }} \sim 2 k T_{\mathrm{e}}$. The fit parameters of these models are shown in Table 5 (lower part).

\section{Discussion}

We carried out a detailed analysis of the Swift J1644+57 spectra observed during outburst decay (2011-2012) and revealed that Swift J1644+57 shows a spectral evolution similar to that seen in Galactic BHs. Specifically, based on Swift, RXTE and Suzaku observations we establish for the Swift J1644+57 spectra $\Gamma$ correlates with the BMC normalization, $N_{\mathrm{BMC}}$ (which is proportional to $\dot{M}$ ) and finally saturates at high values of $\dot{M}$ (see Fig. 8). The index, $\Gamma$, monotonically grows from 1.1 to 1.7 with $\dot{M}$ and then saturates at $\Gamma_{\text {sat }} \sim 1.8$ for high values of $N_{\text {BMC }}$. Previously, Titarchuk \& Zannias (1998) developed the semi-analytical theory of X-ray spectral formation in the converging flow into a $\mathrm{BH}$. They argued that the spectral index of the emergent X-ray spectum saturated at high values of the mass accretion rate (higher than the Eddingtion one). Later analyzing the RXTE data for many BH candidate sources ST09, Titarchuk \& Seifina (2009), Seifina \& Titarchuk (2010), and STS14 demonstrated that this index saturation effect is observed in many Galactic BHs (see e.g., GRS 1915+105 Titarchuk \& Seifina 2009, GX 339-4, GRO J1655-40, 4U 1543-47, H 1743-322, Cyg X1, XTE J1550-564 (ST09) SS 433 Titarchuk \& Seifina 2009). Previously, this scaling method was effectively applied to estimate BH masses of extragalactic black holes (TS16a; Titarchuk \& Seifina 2016b, hereafter TS16b; Sobolewska \& Papadakis 2009; Giacche et al. 2014; Titarchuk \& Seifina 2017, hereafter TS17). Recently the scaling method was successfully implemented to estimate BH masses of two ultraluminous X-ray sources, ESO 243-49 HLX-1 (TS16a) and M101 ULX-1 (TS16b). These findings suggest that BH masses are of the order of $10^{4}$ solar masses in these objects.

The levels of the saturation index are different and presumably depend on the plasma temperature of the converging flow (see Monte Carlo simulations by Laurent \& Titarchuk 1999, 2011). For Swift J1644+57 we establish that the photon index saturates at the relatively low level, $\Gamma_{\text {sat }}$ around 1.7-1.8 (see Fig. 8). This low $\Gamma_{\text {sat }}$ level can be related to the high electron temperature of irradiating plasma $k T_{\mathrm{e}} \sim 40 \mathrm{keV}$, which also is in agreement with the high cutoff energy $E_{\text {cut }} \sim 80 \mathrm{keV}$ detected in the source spectra observed by RXTE (see Figs. 6, 7).

This relatively low $\Gamma_{\text {sat }}$ and consequently, the high $T_{\mathrm{e}}$ value are consistent with each other. It is probable that a large fraction of the mass accretion flow goes out of the disk in the subKeplerian manner. That leads to the strong upscattering of the disk photons in the Compton cloud (transition layer) located between the Keplerian accretion disk and the particle last stable orbit $\left(\sim 3 R_{\mathrm{sch}}\right)$. The geometry of the illumination of the Compton cloud by the soft (disk) photons is shown in TS16a. We note that Very Large Array and Very Long Baseline Array
Table 6. Best-fit parameters of the Swift spectra of Swift $\mathrm{J} 2058+05$ in the $0.3-10 \mathrm{keV}$ range using the following four models: phabs*zphabs*powerlw,phabs*zphabs*zbbody, phabs*zphabs(zbbody+zpowerlw) and phabs*zphabs*bmc.

\begin{tabular}{llll}
\hline \hline & Parameter & 00032004001 & 00032026004 \\
\hline Model & & & \\
\hline phabs & $N_{\mathrm{H}, \mathrm{Gal}}$ & $0.65^{f}$ & $0.65^{f}$ \\
zphabs & $N_{\mathrm{H}, z=1.185}$ & $3.7 \pm 0.8$ & $3.0 \pm 0.1$ \\
zpowerlw & $\Gamma_{\text {zpow }}^{\dagger \dagger}$ & $1.69 \pm 0.08$ & $1.3 \pm 0.1$ \\
& $N_{\text {zpow }}^{\dagger \dagger}$ & $35.1 \pm 0.9$ & $0.41 \pm 0.06$ \\
\hline & $\chi^{2}$ (d.o.f.) & $3.2(967)$ & $1.27(967)$ \\
\hline phabs & $N_{\mathrm{H}, \mathrm{Gal}}$ & $0.65^{f}$ & $0.65^{f}$ \\
zphabs & $N_{\mathrm{H}, z=1.185}$ & $0.2 \pm 0.3$ & $0.6 \pm 0.1$ \\
zbbody & $k T_{z b b}(\mathrm{keV})$ & $3.89 \pm 0.08$ & $1.6 \pm 0.1$ \\
& $N_{z b b}^{\dagger \dagger}$ & $3.7 \pm 0.1$ & $0.007 \pm 0.001$ \\
\hline & $\chi^{2}$ (d.o.f.) & $5.07(967)$ & $1.54(967)$ \\
\hline phabs & $N_{\mathrm{H}, \mathrm{Gal}}$ & $0.65^{f}$ & $0.65^{f}$ \\
zphabs & $N_{\mathrm{H}, z=1.185}$ & $1.9 \pm 0.2$ & $2.3 \pm 0.5$ \\
zbbody & $k T_{z b b}(\mathrm{keV})$ & $5.7 \pm 0.6$ & $1.2 \pm 0.3$ \\
& $N_{z b b}^{\dagger \dagger}$ & $13.6 \pm 0.2$ & $0.3 \pm 0.1$ \\
zpowerlw & $\Gamma_{\text {pow }}^{\dagger \dagger}$ & $1.92 \pm 0.02$ & $1.3 \pm 0.1$ \\
& $N_{\text {pow }}^{\dagger \dagger}$ & $210 \pm 10$ & $15 \pm 9$ \\
\hline & $\chi^{2}$ (d.o.f.) & $1.03(969)$ & $1.17(969)$ \\
\hline phabs & $N_{\mathrm{H}, \mathrm{Gal}}$ & $0.65^{f}$ & $0.65^{f}$ \\
zphabs & $N_{\mathrm{H}, z=1.185}$ & $2.6 \pm 0.1$ & $2.5 \pm 0.2$ \\
bmc ${ }^{z}$ & $\Gamma_{\mathrm{BMC}}$ & $1.78 \pm 0.03$ & $1.5 \pm 0.2$ \\
& $k T_{\mathrm{s}}(\mathrm{eV})$ & $300 \pm 10$ & $270 \pm 7$ \\
& $\log _{\mathrm{b} \text { A }}^{\dagger \dagger}$ & $0.3 \pm 0.1$ & $1.25 \pm 0.6$ \\
& $N_{\mathrm{bmc}}^{\dagger \dagger}$ & $3.1 \pm 0.1$ & $0.09 \pm 0.01$ \\
\hline$\chi^{2}$ (d.o.f.) & $1.04(969)$ & $0.89(969)$ \\
\hline & & & \\
\hline
\end{tabular}

Notes. Errors are given at the $90 \%$ confidence level. ${ }^{(\dagger)}$ The normalization parameters of the blackbody and BMC components are in units of $L_{35}^{\text {soft }} / d_{10}^{2} \mathrm{erg} \mathrm{s}^{-1} \mathrm{kpc}^{-2}$, where $L_{35}^{\text {soft }}$ is the soft photon luminosity in units of $10^{35} \mathrm{erg} \mathrm{s}^{-1}, d_{10}$ is the distance to the source in units of $10 \mathrm{kpc}$, and power-law component is in units of $\mathrm{keV}^{-1} \mathrm{~cm}^{-2} \mathrm{~s}^{-1}$ at $1 \mathrm{keV} . k T_{\mathrm{BB}}$ and $k T_{\mathrm{s}}$ are the temperatures of the blackbody and seed photon components (in $\mathrm{keV}$ and $\mathrm{eV}$ ), respectively. $\Gamma_{\text {pow }}$ and $\Gamma_{\mathrm{BMC}}$ are the indices of the zpowerlw and bmc, respectively. Hereafter superscript ${ }^{z}$ indicates that the cosmological reddening of the spectrum was taken into account $\left(E \rightarrow E(1+z)\right.$ ). Redshift $z$ was fixed to 1.185 (Cenko et al. 2012). $N_{\mathrm{H}, \mathrm{Gal}}$ and $N_{\mathrm{H}, z=1.185}$ are in units of $10^{21} \mathrm{~cm}^{-2}$. ${ }^{(f)}$ Indicates that a parameter was fixed.

radio observations of this X-ray source point to possible outflow (Zauderer et al. 2011, see also Fig. 9). Using the index- $\dot{M}$ correlation found in Swift $\mathrm{J} 1644+57$ we can estimate a BH mass in this source applying scaling of this correlation with those detected in particular GBH sources.

\subsection{Black hole mass estimates}

ST09 (see Sect. 3.2 there) elaborated the scaling method in detail and derived formulae for the BH determination. ST09 (see their Eqs. (4) and (6) ) showed that the disk (soft photon) luminosity is proportional to a dimensionless $\mathrm{BH}$ mass and a mass accretion rate in terms of the solar mass and the critical mass accretion rate, respectively. Moreover, the normalization of the soft photons in the Comptonization BMC model, $N_{\mathrm{BMC}}$ is proportional to the disk (soft photon) luminosity and inversely proportional to $d^{2}$ 


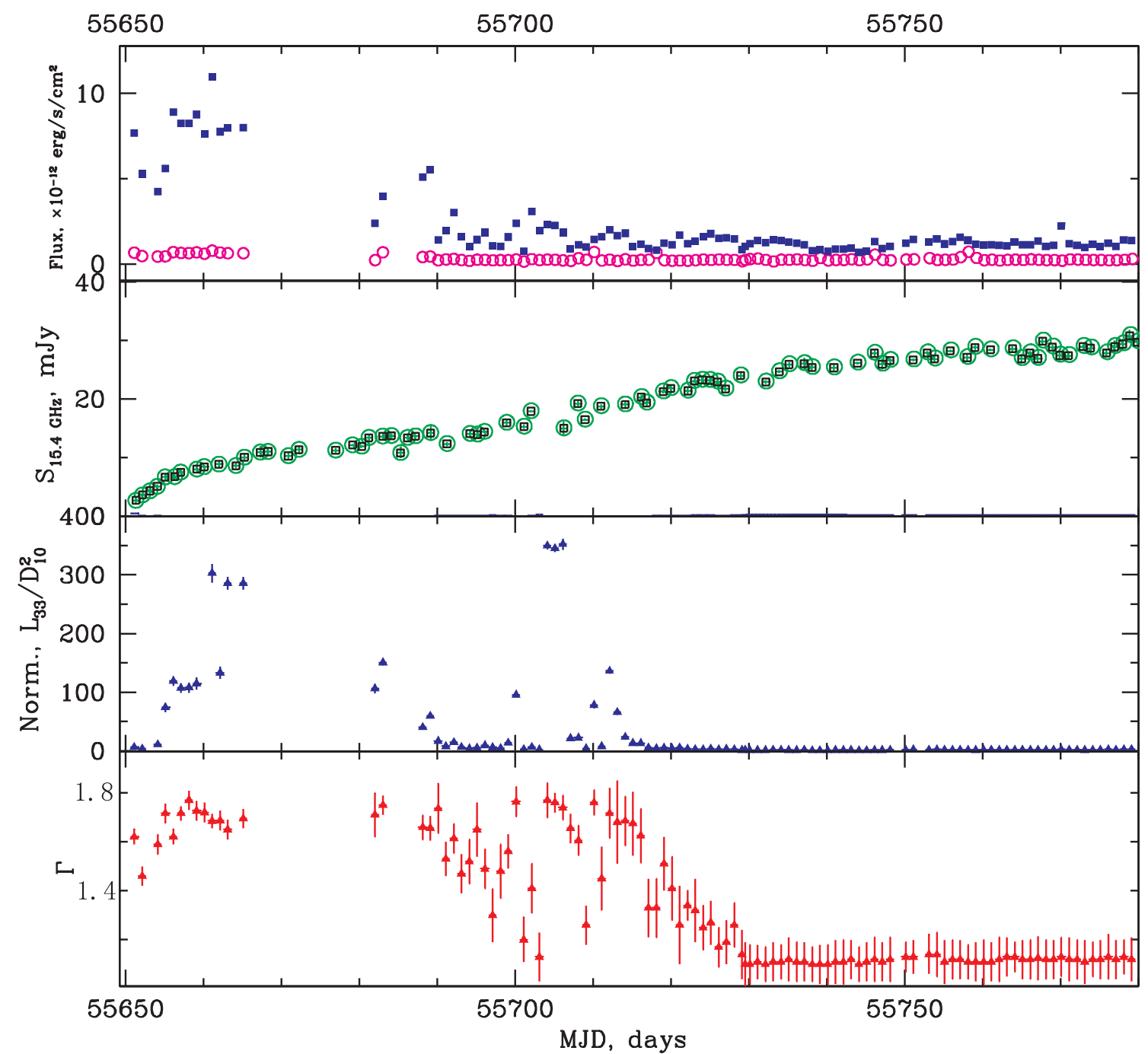

Fig. 9. For Swift J1644+57, from top to bottom: evolutions of the model flux in the soft band $0.3-1.5 \mathrm{keV}$, blue squares, and the hard band 1.5-10 keV, pink circles (Swift/XRT bands); the flux density $S_{15.4 \mathrm{GHz}}$ (green points) at $15.4 \mathrm{GHz}$ (AMI Large Array, Berber et al. 2012), the BMC normalization and the photon index, $\Gamma$ during the 2011 flare decay set (S1, S2).

Table 7. Parameterizations for the target sources, Swift J1644+57 and Swift J2058+05, and reference sources.

\begin{tabular}{lccccc}
\hline \hline Reference source & $\mathcal{A}$ & $\mathcal{B}$ & $\mathcal{D}$ & $x_{\mathrm{tr}}$ & $\beta$ \\
\hline GRO J1655-40 & $1.98 \pm 0.02$ & $0.44 \pm 0.02$ & 1.0 & $0.06 \pm 0.02$ & $1.88 \pm 0.25$ \\
GX 339-4 RISE 2004 & $2.24 \pm 0.01$ & $0.51 \pm 0.02$ & 1.0 & $0.039 \pm 0.002$ & 3.5 \\
4U 1543-37 DECAY 2002 & $2.15 \pm 0.06$ & $0.63 \pm 0.07$ & 1.0 & $0.049 \pm 0.001$ & $0.6 \pm 0.1$ \\
Cyg X-1 & $2.11 \pm 0.06$ & $0.59 \pm 0.08$ & 1.0 & $0.076 \pm 0.003$ & $0.8 \pm 0.1$ \\
\hline Target source & $\mathcal{A}$ & $\mathcal{B}$ & $\mathcal{D}$ & $x_{\mathrm{tr}}\left[\times 10^{-6}\right]$ & $\beta$ \\
\hline Swift J1644+57 & $1.84 \pm 0.09$ & $0.46 \pm 0.08$ & 1.0 & $6.59 \pm 0.09$ & $1.78 \pm 0.08$ \\
Swift J2058+05 & $1.84 \pm 0.09$ & $0.46 \pm 0.08$ & 1.0 & $0.25 \pm 0.08$ & $1.78 \pm 0.08$ \\
\hline
\end{tabular}

(where $d$ is the distance to a particular source). Hence, one can estimate a $\mathrm{BH}$ mass using this relation. The ratio of these normalizations for target and reference sources in the same spectral state is presented by Eq. (7) in ST09.

\subsubsection{Swift J1644+57}

To estimate the BH mass, $M_{\mathrm{BH}}$ of Swift $\mathrm{J} 1644+57$, we chose the galactic BHs, GRO J1655-40, Cyg X-1, 4U 1543-47 and GX 339-4 (see ST09) as the reference sources for which the
BH masses and distances were well established now (see Tables 7,8 ). We note that the $\mathrm{BH}$ masses of these $\mathrm{BHs}$ were also estimated using dynamical methods.

For a BH mass estimate we used $N_{\mathrm{BMC}}$ of the reference sources and the index versus $N_{\mathrm{BMC}}$ correlations for the reference sources, GRO J1655-40, Cyg X-1, 4U 1543-47 and GX 339-4, with that of the target source Swift J1644+57 (see Fig. 12). The value of the index saturation for the Swift J1644+57, $\Gamma_{\text {sat }}^{\mathrm{sw}} \sim 1.78$, is close to $\Gamma_{\text {sat }}^{\text {gro }} \sim 1.98$ of GRO J1655-40 as well as $\Gamma_{\text {sat }}^{\text {cyg }} \sim 2.11$, $\Gamma_{\text {sat }}^{4 u} \sim 2.15$ and $\Gamma_{\text {sat }}^{g x} \sim 2.24$ of Cyg X-1, 4U 1543-47 and 


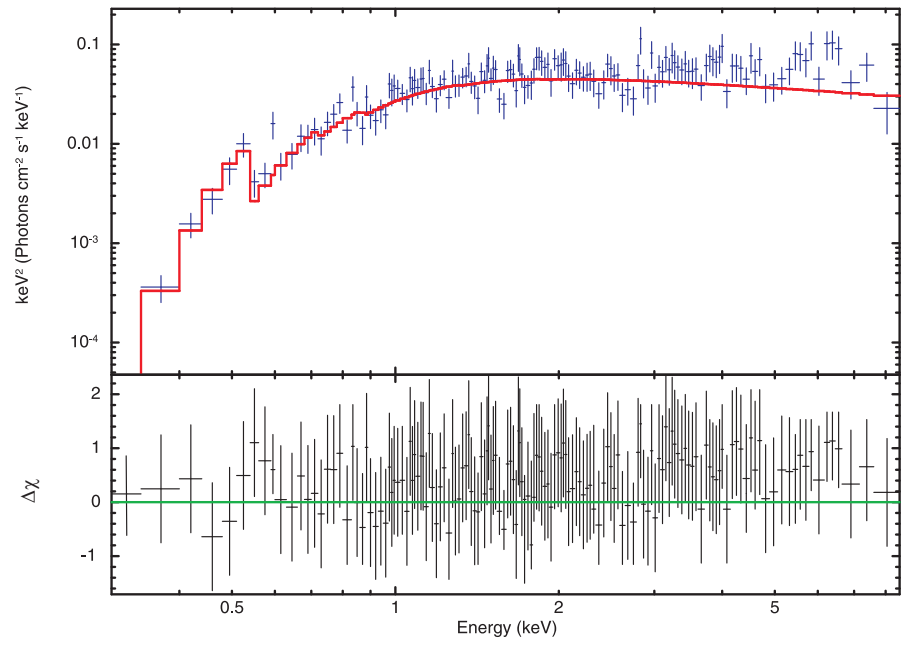

Fig. 10. Best-fit Swift/XRT spectrum in $E F_{\mathrm{E}}$ units during of the intermediate-low hard state transition (top panel) with $\Delta \chi$ (bottom panel) for Swift $\mathbf{J} 2058+05$.

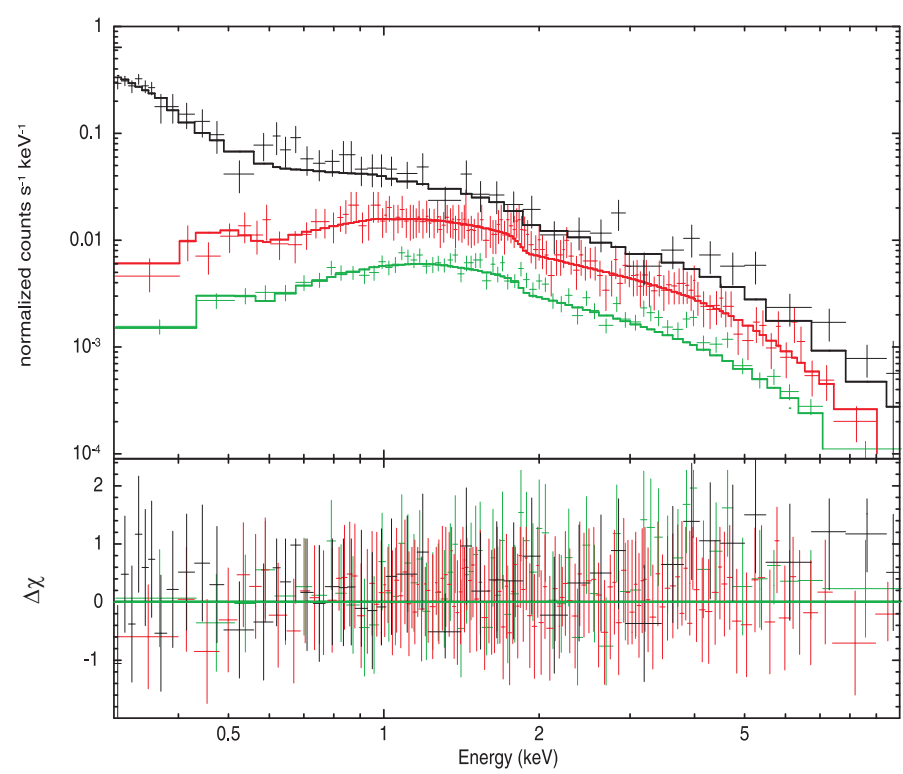

Fig. 11. Three representative Swift/XRT spectra in normalized counts units (top panel) with $\Delta \chi$ (bottom panel) for the LHS (green), IS (red) and HSS (black) spectral states of Swift J2058+05.

GX 339-4, respectively. While such a low index saturation level has never been detected in any Galactic or extragalactic BH source (see i.e., ST09 and TS17), the slopes of $\Gamma-N_{\text {BMC }}$ correlation are the same for both the target and reference sources (see Fig. 12). Hence, it allows us to perform a reliable scaling of this reference source correlation with that of Swift J1644+57. The scaling procedure was made in a similar way to that in TS17; TS16a, b and ST09 where the authors applied an analytical approximation of the $\Gamma\left(N_{\mathrm{bmc}}\right)$ correlation, determined by a function

$$
\begin{aligned}
& \mathcal{F}(x)=\mathcal{A}-(\mathcal{D} \cdot \mathcal{B}) \ln \left\{\exp \left[\left(1.0-\left(x / x_{t r}\right)^{\beta}\right) / \mathcal{D}\right]+1\right\} \\
& \text { with } x=N_{\text {bmc }} .
\end{aligned}
$$

Fitting of the observed correlation by this function $\mathcal{F}(x)$ provides us a number of the best-fit parameters $\mathcal{A}, \mathcal{B}, \mathcal{D}, x_{t r}$, and $\beta$ More detailed description of these parameters is given in TS16a.
One should rely on the same shape of the $\Gamma-N_{\mathrm{bmc}}$ correlations for the target source and those for the reference sources in order to make the $\mathrm{BH}$ mass determination for the target source using the scaling method (ST09). A BH mass, $M_{\mathrm{t}}$ of Swift J1644+57 (target source) can be estimated by shifting the reference source correlation along $N_{\mathrm{bmc}}$-axis to that of the target source (see Fig. 12)

$M_{\mathrm{t}}=M_{\mathrm{r}} \frac{N_{\mathrm{t}}}{N_{\mathrm{r}}}\left(\frac{d_{\mathrm{t}}}{d_{\mathrm{r}}}\right)^{2} f_{G}$,

where the subscripts " $\mathrm{t}$ " and " $\mathrm{r}$ " correspond to the "target" and "reference" sources, respectively and a geometric factor, $f_{G}=(\cos \theta)_{\mathrm{r}} /(\cos \theta)_{\mathrm{t}}$, the inclination angles $\theta_{\mathrm{r}}, \theta_{\mathrm{t}}$ and $d_{\mathrm{r}}, d_{\mathrm{t}}$ are distances to the reference and target sources, respectively (see ST09), values $M_{\mathrm{t}}$ and $M_{\mathrm{r}}$ are in solar masses.

Figure 12 shows the $\Gamma-N_{\mathrm{bmc}}$ correlation for Swift J1644+57 using Swift, Suzaku, and RXTE spectra (see also Tables 4, 5) along with the correlation for the GBHs, GRO 1655-40, GX 339-4, Cyg X-1, and 4U 1543-47.

We use values of $M_{\mathrm{r}}, d_{\mathrm{r}}, d_{\mathrm{t}}$, and $\cos (i)$ from Table 8 and then calculate the lowest limit of the mass, $M_{\mathrm{t}}$ using the best fit value of $N_{\mathrm{t}}$ taken them at the beginning of the index saturation (see Fig. 12) and measured in units of $L_{39} / d_{10}^{2} \mathrm{erg} \mathrm{s}^{-1} \mathrm{kpc}^{-2}$ (see Table 7 for values of the parameters of function $\mathcal{F}\left(N_{\mathrm{t}}\right)$ see Eq. (1)). To determine the distance to Swift J1644+57 we used the formula (for $z<1$ )

$d_{\mathrm{sw}}=z_{\mathrm{sw}} c / H_{0}$,

where the redshift $z_{\mathrm{sw}}=0.354$ for Swift $\mathrm{J} 1644+57$ (see Wright 2006), $H_{0}=70.8 \pm 1.6 \mathrm{~km} \mathrm{~s}^{-1} \mathrm{Mpc}^{-1}$ is the Hubble constant and $c$ is the speed of light. Finally, we obtained that $M_{\mathrm{sw}} \geq$ $7.8 \times 10^{6} M_{\odot}\left(M_{\mathrm{sw}}=M_{\mathrm{t}}\right)$ assuming $d_{\mathrm{sw}} \sim 1.5 \mathrm{Gpc}$. Thus, we obtained a lower limit to the mass due the unknown inclination. We present all these results in Table 8 .

The obtained BH mass estimate is in agreement with a "fundamental plane" estimate, $M_{\mathrm{sw}} \sim 3 \times 10^{6} M_{\odot}$ (see Miller \& Gultekin 2011). In addition, using minimum timescales and the variability method, Liang \& Liu (2003) obtained an estimate of a $\mathrm{BH}$ mass in the interval, $M_{\mathrm{sw}} \sim 10^{7}-10^{9} M_{\odot}$, which is consistent with our $\mathrm{BH}$ mass estimates using the scaling technique.

We derived the bolometric luminosity based on the normalization of the BMC model in the range of $10^{43}-10^{45} \mathrm{erg} / \mathrm{s}$ (assuming isotropic radiation). The relatively high isotropic bolometric luminosity of Swift J1644+57 in the first weeks after the initial outburst is the main argument for a tidal disruption event as an origin of this phenomenon because the host galaxy was not known as an AGN before the flare. The peak bolometric luminosity is in agreement with the derived BH mass using our scaling technique. We note that our peak bolometric luminosity $L_{\mathrm{bol}}^{\text {peak }}$ for Swift $\mathrm{J} 1644+57$ is less than previous estimates of $L_{\text {bol }}^{\text {peak }}$ obtained by different authors (see for example, Komossa 2015; Saxton et al. 2012; Mangano et al. 2016). In particular, Komossa (2015) reported that $L_{\text {bol }}^{\text {peak }} \sim 10^{49} \mathrm{erg} / \mathrm{s}$, which can point to either a larger BH mass $\left(M_{\mathrm{BH}}>10^{9} M_{\odot}\right)$ or to supercritical accretion regime (with $\dot{M}>\dot{M}_{\mathrm{Edd}}$ ). However, for $M_{\mathrm{BH}}$ in the $5 \times 10^{6}-10^{7} M_{\odot}$ range, the disk is expected to have a bolometric disk luminosity in the $(0.5-1) \times 10^{45} \mathrm{erg} / \mathrm{s}$ range which is less than the critical one, $L_{\text {Edd }}$. Therefore, an application of the Comptonization model (BMC) to the spectra of Swift J1644+57 and our scaling method give us the most reliable $\mathrm{BH}$ mass estimate. 


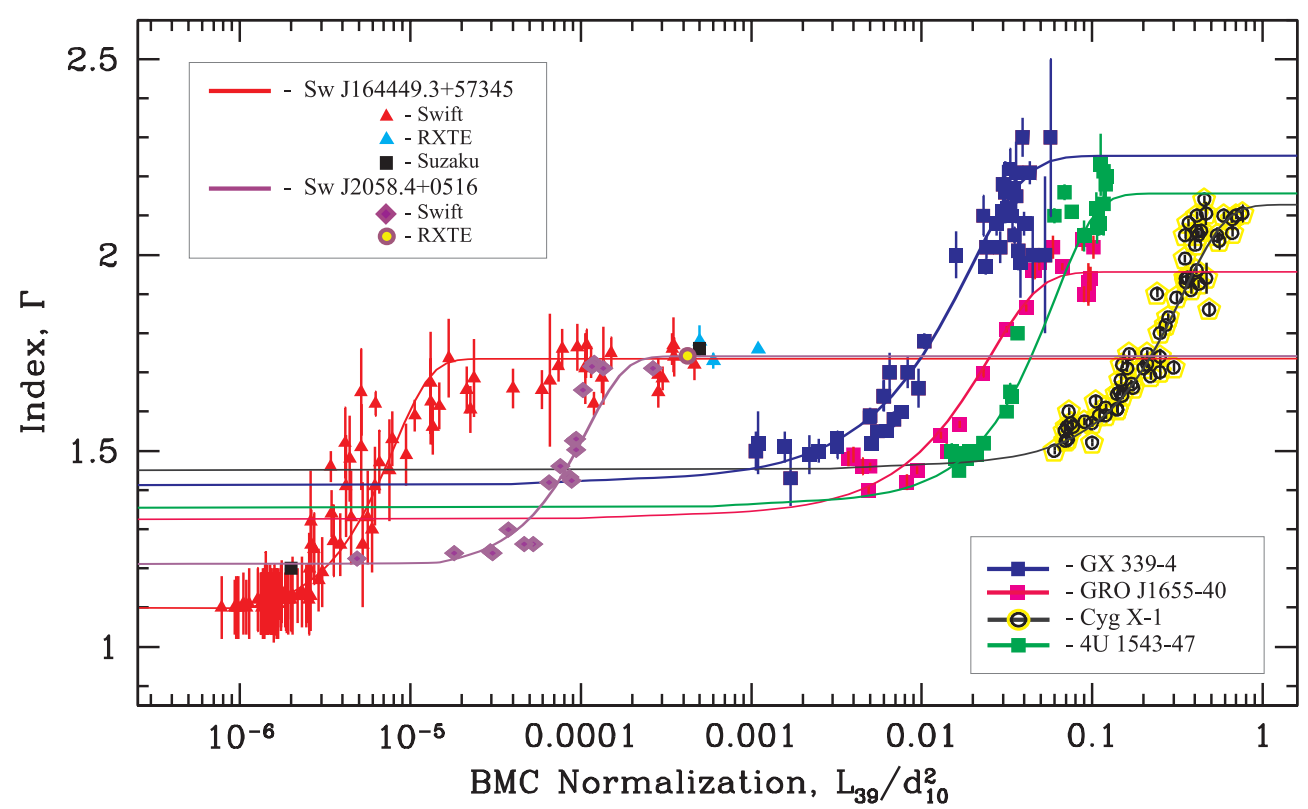

Fig. 12. Scaling of the correlation of the photon index, $\Gamma$ versus the BMC normalization rate (which is proportional to mass accretion rate, $\dot{M}$ ) for Swift J1644+57 (with red/black/bright blue points from Swift/Suzaku/RXTE data) and Swift J2058+05 (with violet diamonds/circle from Swift/RXTE data), and that for GRO J1655-40 (with pink squares, ST09), GX 339-4 (with blue squares, ST09), Cyg X-1 (with black circles, ST09) and 4U 1543-47 (with green squares, ST09) as a function of BMC normalization.

Table 8. Esimates of a BH mass and a distance value for the target sources, Swift J1644+57 and Swift J2058+05, and those for reference sources.

\begin{tabular}{llllllc}
\hline \hline Source & $M_{\text {dyn }}^{\dagger}\left(M_{\odot}\right)$ & $i_{\text {orb }}^{\dagger}(\mathrm{deg})$ & $d^{\dagger \dagger}(\mathrm{kpc})$ & $M_{\text {fund.plane }}\left(M_{\odot}\right)$ & $M_{\text {min.timescale }}\left(M_{\odot}\right)$ & $M_{\text {scal }}\left(M_{\odot}\right)$ \\
\hline GX 339-4 & $>6 c^{(1,2)}$ & $\ldots$ & $7.5 \pm 1.6^{(2)}$ & $\ldots$ & $\ldots$ & $5.7 \pm 0.8^{\dagger \dagger \dagger}$ \\
4U 1543-47(3,4) & $9.4 \pm 1.0$ & $20.7 \pm 1.5$ & $7.5 \pm 1.0$ & $\ldots$ & $\ldots .4 \pm 1.4^{\dagger \dagger}$ \\
Cyg X-1 & $6.8-13.3$ & $35 \pm 5$ & $2.5 \pm 0.3$ & $\ldots$ & $\ldots$ & $7.9 \pm 1.0^{\dagger \dagger \dagger}$ \\
GRO J1655-40 & $6.3 \pm 0.3^{(7,8)}$ & $70 \pm 1^{(7,8)}$ & $3.2 \pm 0.2^{(9)}$ & $\ldots$ & $\ldots$ & $\ldots$ \\
Swift J1644+57 & $\ldots$ & $\ldots$ & $\sim 1.5 \times 10^{6}$ & $\sim 3 \times 10^{6}$ & $\sim 10^{7}-10^{9}$ & $\geq 7.8 \times 10^{6}$ \\
Swift J2058+05(14,15,16) & $\ldots$ & $\ldots$ & $\sim 3.7 \times 10^{6}$ & $\ldots$ & $\sim 5 \times 10^{7}$ & $\geq 2 \times 10^{7}$ \\
\hline
\end{tabular}

Notes. ${ }^{(\dagger)}$ Dynamically determined BH mass and system inclination angle; ${ }^{(\dagger)}$ Source distance found in the literature. For Swift J2058+0516 the distance is estimated using the redshift $z$ with taking into account the cosmological effects for $z>1$ (see Wright $2006^{1}$ ). ${ }^{(\dagger \dagger+)}$ Scaling value found by ST09.

References. (1) Munoz-Darias et al. (2008); (2) Hynes et al. (2004); (3) Orosz (2003); (4) Park et al. (2004); (5) Herrero et al. (1995); (6) Ninkov et al. (1998); (7) Green et al. (2001); (8) Hjellming \& Rupen (1995); (9) Jonker \& Nelemans (2004); (10) Miller \& Gultekin (2011); (11) Bloom et al. (2011); (12) Burrows et al. (2011); (13) Levan et al. (2011); (14) Komossa (2015); (15) Cenko et al. (2012); (16) Pasham et al. (2015).

Indeed, the inferred luminosity is close to the Eddington limit for a $10^{7}$ solar masses $\mathrm{BH}, L_{\mathrm{Edd}}=4 \pi c G M_{\mathrm{BH}} / \sigma_{\mathrm{T}} \sim$ $1.4 \times 10^{45} \mathrm{erg} / \mathrm{s}$. The relatively high electron temperatures, $k T_{\mathrm{e}} \sim 34 \mathrm{keV}$ (see Table 5) are obtained using CompTB model and the RXTE data for Swift J1644+57 spectra (see Sect. 3.4 and Figs. 6, 7). For such a high $T_{\mathrm{e}}$ the effective scattering crosssection, $\langle\sigma\rangle$ is less than the Thomson one, $\sigma_{\mathrm{T}}$ (see, for example Titarchuk et al. 2014) and consequently, the critical luminosity is higher than the Eddington one. But the temperature-corrected critical luminosity in the case of Swift J1644+57 is higher than $L_{\mathrm{Edd}}$ of $1.4 \times 10^{45} \mathrm{erg} / \mathrm{s}$ and thus, the observed luminosity from this TDE source is actually a few times less than the critical one.

\subsubsection{Swift J2058+05}

To estimate a BH mass of Swift J2058+05, we chose the galactic BH, GRO J1655-40 (see ST09), Cyg X-1, GX 339-4, 4U 154347 and the TDE source, Swift J1644+57 (see Sect. 4.1.1) as the

\footnotetext{
1 http://www. astro.ucla.edu/ wright/CosmoCalc.html
}

reference sources for which the $\mathrm{BH}$ masses and distances were established now (see Tables 7, 8).

For a BH mass estimate we used $N_{\text {BMC }}$ of the reference sources and the index versus $N_{\mathrm{BMC}}$ correlations for the abovementioned reference sources, with that of the target source, Swift J2058+05 (see Fig. 12). The value of the index saturation for the Swift J2058+05, $\Gamma_{\mathrm{sat}}^{\mathrm{sw2}} \sim 1.76$, is close to $\Gamma_{\mathrm{sat}}^{\mathrm{sw1}} \sim 1.78$ for Swift J1644+57, as well as to $\Gamma_{\text {sat }}^{\text {gro }} \sim 1.98$ for GRO J1655-40, as well as $\Gamma_{\text {sat }}^{\mathrm{cyg}} \sim 2.11, \Gamma_{\text {sat }}^{4 u} \sim 2.15$ and $\Gamma_{\text {sat }}^{g x} \sim 2.24$ of Cyg X-1, $4 \mathrm{U} 1543-47$ and GX 339-4, respectively. While such low index saturation levels (in Swift J2058+05 and Swift J1644+57) have never been found in any Galactic or extragalactic BH source (see ST09 and Sect. 4.1.1).

The slopes of $\Gamma-N_{\mathrm{BMC}}$ correlation are the same for all the target and reference sources (see Fig. 12). Therefore, it allows us to perform a reliable scaling of these reference source correlation with that of Swift $\mathbf{J} 2058+05$. The scaling procedure was made in a similar way as Sect. 4.1.2 and in TS17, TS16a, Titarchuk \& Seifina (2016b, hereafter TS16b) and ST09 where the authors 
applied an analytical approximation of the $\Gamma\left(N_{\mathrm{bmc}}\right)$ correlation (see Eq. (1)).

A BH mass, $M_{\mathrm{t}}$ of Swift $\mathrm{J} 2058+05$ (target source) can be estimated using by the same method as that for Swift J1644+57. We shift the reference source correlations along $N_{\text {bmc }}$-axis to that of the target source (see Fig. 12 and Eq. (2)). Figure 12 shows the $\Gamma-N_{\text {bmc }}$ correlation for Swift J2058+05 using Swift, and RXTE (see also Tables 5, 6 along with the correlation for the GRO 1655-40 and Swift J1644+57 using Swift, Suzaku, and RXTE spectra. We use values of $M_{\mathrm{r}}, d_{\mathrm{r}}, d_{\mathrm{t}}$, and $\cos (i)$ from Table 8 and then calculate the lowest limit of the mass, $M_{\mathrm{t}}$ using the best fit value of $N_{\mathrm{t}}$ taken at the beginning of the index saturation (see Fig. 12).

To determine the distance to Swift J2058+05 we use the formula (for $z>1$ )

$d_{2058} \approx \frac{2 c}{H_{0}}\left[1-\frac{1}{\sqrt{z_{2058}}}\right]$,

where the redshift $z_{2058}=1.185$ for Swift $\mathbf{J} 2058+05$ (Wright 2006). Thus, we obtain that $M_{2058} \geq 2 \times 10^{7} M_{\odot}\left(M_{\mathrm{sw} 2}=M_{\mathrm{t}}\right)$ assuming $d_{\mathrm{sw} 2} \sim 3.7 \mathrm{Gpc}$. Thus, we estimated a lower limit of the $\mathrm{BH}$ mass due to the unknown inclination. We present all these results in Table 8.

The obtained $\mathrm{BH}$ mass estimate is in agreement with the mass limits derived based on the X-ray turnoff $\left(10^{4} M_{\odot} \leq\right.$ $M_{\mathrm{BH}} \leq 2 \times 10^{6} M_{\odot}$, Pasham et al. 2015) as well as using the method of estimate of a $\mathrm{BH}$ mass upper limit applying the X-ray variability timescale, $5 \times 10^{7} M_{\odot}$ (see Pasham et al. 2015). Furthermore, Pasham et al. (2015) assumed that the TDE source optical flux is dominated by the host galaxy and they constrained the BH mass of the central SMBH using the well-known bulge luminosity as a function of BH mass relations (e.g., Lauer et al. 2007). As a result they inferred the SMBH mass of about $M_{\mathrm{BH}} \sim 3 \times 10^{7} M_{\odot}$, which is consistent with our BH mass estimates using the scaling technique.

We derived the bolometric luminosity, based on the normalization of the BMC model, in the range of $10^{44}-10^{45} \mathrm{erg} / \mathrm{s}$ (assuming isotropic radiation). The relatively high isotropic bolometric luminosity of Swift J2058+05 in the first weeks after the initial outburst is the main argument for a TDE as an origin of this phenomenon because the host galaxy was not known as an AGN before the flare. In particular, Pasham et al. (2015; see the top panel in their Fig. 1) and Komossa (2015) speculated that $L_{\text {bol }}^{\text {peak }} \sim 3 \times 10^{47} \mathrm{erg} / \mathrm{s}$, and they estimated that the BH mass, $M_{\mathrm{BH}}>10^{7} M_{\odot}$ which is similar to our scaling technique estimate (our inferred luminosity at the maximum for Swift $\mathrm{J} 2058+05$ is close to the Eddington limit for a $10^{7}$ solar masses BH, see Sect. 4.1.1).

The relatively low electron temperatures, $k T_{\mathrm{e}} \sim 4-10 \mathrm{keV}$ (see also Table 5; lower part) are obtained using CompTB model and the RXTE data for Swift J2058+05 spectra (see Sect. 3.2.2) For such a low $T_{\mathrm{e}}$ the effective scattering crosssection, $\langle\sigma\rangle$ is about of the Thomson one, $\sigma_{\mathrm{T}}$ (see, for example Titarchuk et al. 2014) and consequently, the critical luminosity is close the Eddington one.

\subsection{Comparison of spectral and timing characteristics of TDE sources Swift J2058+05 and Swift J1644+57}

Because of the many similarities between Swift J2058+08 and Swift J1644+57, Cenko et al. (2012) suggested a similar outburst mechanism, consistent with multi-wavelength follow-up observations (Pasham et al. 2015). Below, we present a comparison of these sources in terms of spectral and timing properties to further reveal the similarities and the differences between TDE sources Swift J2058+05 and Swift J1644+57.

\subsubsection{Saturation levels of the photon index}

The TDE sources Swift J2058+05 and Swift J1644+57 demonstrate a similar behavior of the photon index versus mass accretion rate (or our BMC normalization). The saturations of the photon index occurs at the same saturation level $\Gamma_{\text {sat }} \sim 1.8$ for both of these sources.

\subsubsection{Difference of the electron temperature ranges in Swift J2058+05 and Swift J1644+57}

A comparison of the best-fit spectral parameters for these two TDE sources shows that the ranges of seed (disk) temperatures are similar for both of these objects, namely, $k T_{\mathrm{s}}=100-400 \mathrm{eV}$ (see Tables 4, 6). On the other hand, values of the electron temperature $k T_{\mathrm{e}}$ are quite different. These values, $k T_{\mathrm{e}}$ vary in a wide range up to $k T_{\mathrm{e}}=35 \mathrm{keV}$ for Swift $\mathrm{J} 1644+57$, while for Swift $\mathbf{J} 2058+05 k T_{\mathrm{e}}$ they are between $4 \mathrm{keV}$ and $10 \mathrm{keV}$. The reason for this difference of temperature ranges is not so obvious but we can suggest that the soft (disk) photon emission is stronger in Swift $\mathrm{J} 2058+05$ than that in the case of Swift J1644+57 and thus, $k T_{\mathrm{e}}$ are lower in Swift $\mathbf{J} 2058+05$ than that in Swift $\mathbf{J} 1644+57$.

\subsubsection{Similarity of timing characteristics during decay phases in Swift J2058+05 and Swift J1644+57}

Similarly to Swift J1644+57, the lightcurve of Swift J2058+05 shows an abrupt drop during 250-300 days (see, e.g., Cenko et al. 2012; Pasham et al. 2015) while secular decline is described by different decay rates. More specifically, the decline in flux of Swift J1644+57 is consistent with the $t^{-1.5-1.67}$ (Bloom et al. 2011; Levan et al. 2011; Mangano et al. 2016), while that for Swift J2058+05 is consistent with $t^{-2.2}$ (Cenko et al. 2012). The reason for this difference for flux decline rates is not clear up to now but it can be associated with different outflow plasma conditions in these two sources. The count rate in Swift J2058+05 decreases by a factor of 150 (see Fig. 2) which is comparable with that in Swift J1644+57, a factor of $\sim 100$ decline, (see Levan \& Tanvir 2012; Sbarufatti et al. 2012; Zauderer et al. 2013).

Notably, in both of these sources, the X-ray dimming occurs on a similar timescale after disruption. In the case of Swift J1644+57, Zauderer et al. (2013) argued that this sudden decrease in the flux is caused by the drop of an accretion flow from a super-Eddington to a sub-Eddington rates. Using numerical simulations by Evans \& Kochanek (1989) and De Colle et al. (2012) one can support this scenario. We note that applying the abrupt flux change, Pasham et al. (2015) constrained the black hole mass $M_{\mathrm{BH}}$ in the range of $10^{4} M_{\odot}$ to $2 \times 10^{6} M_{\odot}$ in Swift J2058+05.

\subsubsection{Comparison of spectral evolution as a function of the normalization for Swift J2058+05 and Swift J1644+57}

We can also compare spectral parameter evolution for Swift J2058+05 and Swift J1644+57 using the BMC normalization. We note that the distances to these sources are different (see Table 8). Specifically, for Swift J2058+05 the distance is about 
3.7 Gpc, whereas for Swift J1644+57 it is 1.5 Gpc. In Fig. 12, we show correlations of BMC normalization, presumably proportional to mass accretion rate, and the photon index $\Gamma$ for these two TDE sources. Swift J1644+57 demonstrates a wider range of BMC normalization with a longer saturation part (by a factor of two higher than that for Swift $\mathrm{J} 2058+05)$, while $k T_{\mathrm{s}}$ are almost the same for both sources $(100-400 \mathrm{eV})$.

\section{Conclusions}

A stellar tidal disruption event (TDE) presents a new chance to estimate the mass of accreting supermassive black holes. The flare events occur after disruption of a star's orbit at about ten of Schwarzschild radii from the central supermassive BH. A large amount of gas is suddenly injected close to a $\mathrm{BH}$ event horizon, as this stellar debris gets accreted (Giannios \& Metzger 2011; van Velzen et al. 2011).

We find the transition from the high to low states observed in Swift J1644+57 and Swift J2058+05 during decay outburst phase using the set of Swift, Suzaku, and RXTE observations. We reveal a validity of the fits of the observed spectra using the BMC model for all observations, independently of the spectral state of the source.

We investigated the X-ray outburst properties of TDEs during the outbursts using of hardness-intensity diagrams and the index-normalization (or $\dot{M}$ ) correlation, which were similar to those in Galactic BHs. In particular, we find that Swift J1644+57 approximately follows the $\Gamma-\dot{M}$ correlation previously obtained for the Galactic BHs, GRO J1655-40, GX 339-4, Cyg X-1 and 4U 1543-47 (see Fig. 12). The photon index of Swift J1644+57 spectrum is in the range of $\Gamma=1.1-1.8$. We also find that Swift $\mathbf{J} 2058+05$ approximately follows the $\Gamma-\dot{M}$ correlation obtained for the Galactic BH, GRO J1655-40, and Swift J1644+57. The photon index of the Swift $\mathbf{J} 2058+05$ spectra is in the range of $\Gamma=1.2-1.8$.

We used the observed index-mass accretion rate correlation to estimate $M_{\mathrm{BH}}$ in Swift J1644+57 and Swift 2058+05. This scaling method was successfully implemented to find $\mathrm{BH}$ masses of Galactic (e.g., ST09, STS14) and extragalactic black holes (TS16a,b; Sobolewska \& Papadakis 2009; Giacche et al. 2014; TS17). We find that the compact objects, Swift J1644+57 and Swift J2058+05 are likely to be supermassive BHs with $M_{\mathrm{BH}} \geq 7 \times 10^{7} M_{\odot}$ and $\geq 2 \times 10^{7} M_{\odot}$, correspondingly.

Acknowledgements. This research was done using the data supplied by the UK Swift Science Data Centre at the University of Leicester. We appreciate Cristiano Guidorzi who raised a fair question on the origin of the TDE sources. Partic ularly, we also acknowledge extensive discussion and critical points by Sergio Campana on the paper content, Pascal Chardonnet for useful discussions and comments and Partick Aurenche for editing of the paper. Finally, we recognize the thorough analysis of the paper by an anonymous referee.

\section{References}

Aliu, E., Arlen, T., Aune, T., et al. 2011, ApJ, 738, L30

Aleksic, J., Antonelli, L. A., Antoranz, P., et al. 2013, A\&A, 552, A112

Belloni, T., \& Motta, S. E. 2016, Astrophysics of Black Holes (Berlin Heidelberg: Springer-Verlag), Astrophys. Space Sci. Lib., 440, 61

Belloni, T., Parolin, I., Del Santo, M., et al. 2006, MNRAS, 367, 1113

Bennert V. N., Auger M. W., Treu T., Woo J.-H., \& Malkan M. A. 2011, ApJ, 726,59
Berger, E., Zauderer, A., Pooley, G. G., et al. 2012, ApJ, 748, 36 Bloom J. S., Giannios, D., Metzger, B. D., et al. 2011, Science, 333, 203 Burrows, D. N., Kennea, J. A., Ghisellini, G., et al. 2011, Nature, 476, 421 Castro-Tirado, A. J., Gómez, J. L., Agudo, I., et al. 2013, EPJ Web Conf., 61, 01003

Cendes, Y., Wijers, R. A. M. J., Swinbank, J. D., et al. 2014, ArXiv e-prints [arXiv: 1412.3986]

Cenko, S. B., Krimm, H. A., Horesh, A., et al. 2012, ApJ, 753, 77

De Colle, F., Guillochon, J., Naiman, J., \& Ramirez-Ruiz, E. 2012, ApJ, 760, 103

Evans, C. R., \& Kochanek, C. S. 1989, ApJ, 346, L13

Evans, P. A., Beardmore, A. P., Page, K. L., et al. 2007, A\&A, 469, 379

Evans, P. A., Beardmore, A. P., Page, K. L., et al. 2009, MNRAS, 397, 1177

Farinelli, R., Titarchuk, L., Paizis, A., \& Frontera, F. 2008, ApJ, 680, 602 (F08)

Giacche, S., Gili, R., \& Titarchuk, L. 2014, A\&A, 562, A44

Giannios, D., \& Metzger, B. D. 2011, MNRAS, 416, 2102

González-Rodriguez, A., Castro-Tirado, A. J., Guerrero, M. A., \& Castellón, A.

2014, in Rev. Mex. Astron. Astrofis. (Serie de Conferencias), 45, 73

Graham, A. W. 2012, ApJ, 746, 113

Greene, J., Bailyn, C. D., \& Orosz, J. A. 2001, ApJ, 554, 1290

Herrero, A., Kudritzki, R. P., Gabler, R., Vilchez, J. M., \& Gabler, A. 1995, A\&A, 297, 556

Hjellming, R. M., \& Rupen, M. P. 1995, Nature, 375, 464

Homan, J., Wijnands, R., van der Klis, M., et al. 2001, ApJS, 132, 377

Jahnke, K., \& Maccio, A. V. 2011, ApJ, 734, 92

Jonker, P. G., \& Nelemans, G. 2004, MNRAS, 354, 355

Kalberla, P. M. W., Burton, W. B., Hartmann, D., et al. 2005, A\&A, 440, 775

Kennea, J. A., Romano, P., Krimm, H. A., et al. 2011, ATel, 3250, 1

Komossa, S. 2015, J. High Energy Astrophys., 7, 148

Krimm, H. A., Tomsick, J. A., Markwardt, C. B., et al. 2011, ApJ, 735, 104

Lauer, T. R., Faber, S. M., Richstone, D., et al. 2007, ApJ, 662, 808

Laurent, P., \& Titarchuk, L. 1999, ApJ, 511, 289 (LT99)

Laurent, P., \& Titarchuk, L. 2011, ApJ, 727, L34

Levan, A. J., Tanvir, N. R., Cenko, S. B., et al. 2011, Science, 333, 199

Liang, E. W., \& Liu, H. T. 2003, MNRAS, 340, 632

Mangano, V., Burrows, D. N., Sbarufatti, B., \& Cannizzo, J. K. 2016, ApJ, 817, 103

Migliari, S., \& Fender, R. P. 2006, MNRAS, 366, 79

Miller, J. M., \& Gultekin, K. 2011, ApJ, 738, L13

Munoz-Darias, T., Casares, J., \& Martnez-Pais, I. G. 2008, MNRAS, 385, 2205

Munoz-Darias, T., Fender, R. P., Motta, S. E., \& Belloni, T. M. 2014, MNRAS, 443, 3270

Ninkov, Z., Walker, G. A. H., \& Yang, S. 1987, ApJ, 321, 425

Park, S. Q., Miller, J. M., McClintock, J. E., et al. 2004, ApJ, 610, 378

Park, T., Kashyap, V. L., Siemiginowska, A., et al. 2006, ApJ, 652, 610

Pasham, D. R., Cenko, S. B., Levan, A. J., et al. 2015, ApJ, 805, 68

Rees, M. J. 1988, Nature, 333, 523

Reis, R. C., Miller, J. M., Reynolds, M. T., et al. 2012, Science, 337, 949

Saxton, C. J., Soria, R., Wu, K., \& Kuin, N. P. M. 2012, MNRAS, 422, 1652

Sbarufatti, B., Burrows, D. N., Gehrels, N., \& Kennea, J. A. 2012, ATel, 4398, 1

Seifina, E., \& Titarchuk, L. 2010, ApJ, 722, 586

Shaposhnikov, N., \& Titarchuk, L. 2006, ApJ, 643, 1098 (ST06)

Shaposhnikov, N., \& Titarchuk, L. 2009, ApJ, 699, 453 (ST09)

Shrader, C. R., Titarchuk, L., \& Shaposhnikov, N. 2010, ApJ, 718, 488

Silk, J., \& Rees, M. J. 1998, A\&A, 331, L1

Sobolewska, M. A., \& Papadakis, I. E. 2009, MNRAS, 399, 1997

Titarchuk, L., \& Seifina, E. 2009, ApJ, 706, 1463

Titarchuk, L., \& Seifina, E. 2016a, A\&A, 595, A101 (TS16a)

Titarchuk, L., \& Seifina, E. 2016b, A\&A, 585, A94 (TS16b)

Titarchuk, L., \& Seifina, E. 2017, A\&A, 602, A113

Titarchuk, L., \& Zannias, T. 1998, ApJ, 499, 315 (TZ98)

Titarchuk, L., Mastichiadis, A., \& Kylafis, N. D. 1997, ApJ, 487, 834

Titarchuk, L. Seifina, E., \& Shrader, C. 2014, ApJ, 789, 98

Usui, R., \& Kawai, N. 2015, Proc. Int. Symp. Nanoscience and Quantum Physics 2012, JPS Conf. Peoc., 4, 01003

van Velzen, S., Körding, E., \& Falcke, H. 2011, MNRAS, 417, L51

Wiersema, K., van der Horst, A. J., Levan, A. J., et al. 2012, MNRAS, 421, 1942

Wright, E. L. 2006, PASP, 118, 1711

Zauderer, B. A., Berger, E., Soderberg, A. M., et al. 2011, Nature, 476, 425

Zauderer, B. A., Berger, E., Margutti, R., et al. 2013, ApJ, 767, 152 